\title{
Reduction of Homogeneous Riemannian Structures
}

\author{
M. Castrillón López \\ ICMAT (CSIC-UAM-UC3M-UCM) \\ Departamento de Geometría y Topología \\ Facultad de Matemáticas, Universidad Complutense de Madrid \\ 28040 Madrid, Spain \\ Ignacio Luján \\ Departamento de Geometría y Topología \\ Facultad de Matemáticas, Universidad Complutense de Madrid \\ 28040 Madrid, Spain
}

\begin{abstract}
The goal of this article is the study of homogeneous Riemannian structure tensors within the framework of reduction under a group $H$ of isometries. In a first result, $H$ is a normal subgroup of the group of symmetries associated to the reducing tensor $\bar{S}$. The situation when $H$ is any group acting freely is analyzed in a second result. The invariant classes of homogeneous tensors are also investigated when reduction is performed. It turns out that the geometry of the fibres is involved in the preservation of some of them. Some classical examples illustrate the theory. Finally, the reduction procedure is applied to fiberings of almost contact manifolds over almost Hermitian manifolds. If the structure is moreover Sasakian, the obtained reduced tensor is homogeneous Kähler.
\end{abstract}

\section{Introduction}

Since their introduction 2, homogeneous structure tensors has proved to be a powerful tool in the study of homogeneous Riemannian manifolds. Their nature is twofold. On one hand, they belong to the tensor algebra. In particular, representation theory techniques classify them into eight different invariant classes with respect to a convenient action of the orthogonal group. On the other hand, homogeneous tensors satisfy a system of partial differential equations (Ambrose-Singer equations). Many works in the literature combine these

MSC2010: Primary 53C30, Secundary 53D35, 22F30.

Key words and phrases: Connection, contact and Hermitian structures, homogeneous structures, fibre bundle, reduction. 
aspects to provide geometric properties of the underlying Riemannian manifold. The first characterizations were given to hyperbolic space and naturally reductive spaces ([18]). These techniques were subsequently generalized to Riemannian manifolds with special holonomy by many authors (for example, [1, [4], 6, 7], 11]). It is interesting to point out that there is not a bijection between tensors and possible groups acting isometrically and transitively. A same tensor can be defined by two different groups and a same group can provide different tensors. In this context, it is remarkable how little is known about all homogeneous structures and tensors for even well-known spaces. There is still much work to do.

Manifolds endowed with symmetries are relevant in many situations. In particular, symmetries represent a classical tool in reduction schemes intimately related with different topics as systems of differential equations, variational principles, symplectic or other geometric structures, etc. In particular, reduction is recurrently applied in homogeneous manifolds. The goal of this article is the study of the behaviour of homogeneous tensors by reduction under subgroups of the group of isometries. In particular, this gives rise to new homogeneous tensors in the orbit space of the action. Additionally, the reduction process reveals and sheds light to some previously known properties of some homogeneous structures. Finally, the reduction technique opens a reverse way to get new homogeneous tensors in the unreduced space from tensors in the orbit space.

The outline of the paper is as follows. In section 2 we recall basic definitions on homogeneous structure tensors and its classification. Moreover, the model for reduction will be a Riemannian principal bundle $\bar{M} \rightarrow M$, endowed with the compatible connection defined as the orthogonal complements to the fibres. This connection is ubiquitously used for reduction schemes in Mechanics (see for example, 14, 15) where it is called the mechanical connection. Section 3 begins with reduction of homogeneous tensors $\bar{S}$ in $\bar{M}$ by the action under a normal subgroup $H$ of the group of symmetries $\bar{G}$ associated to $\bar{S}$ (Theorem 3.4). The space of all tensors $\bar{S}$ projecting to a same tensor $S$ in $M=\bar{M} / H$ is also determined. The expression of the reduced tensors leads to a generalization of the reduction result (Theorem 3.7) to the case where $\bar{S}$ is not explicitly associated to a precise group $\bar{G}$. For example, this is the case of non-simply connected or uncomplete manifolds where the existence of homogenous tensors still provides interesting geometric properties. Without the presence of the group $\bar{G}$, the normality of the structure group $H$ of the bundle $\bar{M} \rightarrow M$ needs to be replaced by a suitable differential condition on the mechanical connection. Finally, the behaviour of the classification of homogeneous tensors under the reduction process is analyzed. It is interesting to point out that the geometry of the orbits of the $H$-action is involved in some of the classes in this classification. Section 4 provides many examples of the main results of the article. In particular, they explore the possible scenarios with respect to the classes when reduction is performed. Section 5 applies the reduction Theorem to fiberings of almost contact manifolds over almost Hermitian manifolds ([16]). It turns out that the differential condition on the mechanical connection is automatically satisfied for homogeneous almost contact or Sasakian tensors. Hence they 
project to homogeneous almost Hermitian or Kähler tensors in a natural way. This is connected with other constructions found in the literature (see [8]).

\section{Preliminares}

\subsection{Homogeneous Riemannian structures}

Let $(M, g)$ be a connected Riemannian manifold of dimension $n$. Let $\nabla$ be the Levi-Civita connection of $g$ and $R$ its curvature tensor with the convention

$$
R_{X Y} Z=\nabla_{X} \nabla_{Y} Z-\nabla_{Y} \nabla_{X} Z-\nabla_{[X, Y]} Z
$$

A homogeneous Riemannian structure on $(M, g)$ is a $(1,2)$-tensor field $S$ satisfying the so called Ambrose-Singer equations

$$
\widetilde{\nabla} g=0, \quad \widetilde{\nabla} R=0, \quad \widetilde{\nabla} S=0,
$$

where $\widetilde{\nabla}=\nabla-S[18$. We will also denote by $S$ the associated $(0,3)$-tensor field obtained by lowering the contravariant index, $S_{X Y Z}=g\left(S_{X} Y, Z\right)$.

We now suppose that $(M, g)$ is homogeneous Riemannian. Let $G$ be a connected Lie group with Lie algebra $\mathfrak{g}$ acting effectively and transitively on $M$ by isometries. And let $K$ be the isotropy group at a point $x \in M$ with Lie algebra $\mathfrak{k}$. A decomposition $\mathfrak{g}=\mathfrak{m} \oplus \mathfrak{k}$ is said to be a reductive decomposition of $\mathfrak{g}$ if $A d(K)(\mathfrak{m}) \subset \mathfrak{m}$. Let $\mu$ be the infinitesimal action of $\mathfrak{g}$ at the point $x$, that is

$$
\begin{aligned}
\mu: \mathfrak{g} & \rightarrow T_{x} M \\
\xi & \left.\mapsto \frac{d}{d t}\right|_{t=0} \Phi_{\exp (t \xi)}(x)
\end{aligned}
$$

where $\Phi_{a}$ denotes the action of an element $a \in G$. Then for all $k \in K$ the following diagram is commutative

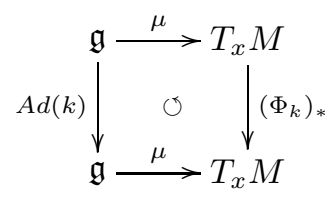

The restriction of $\mu$ to $\mathfrak{m}$ gives an isomorphism $\mu: \mathfrak{m} \rightarrow T_{x} M$, and the canonical connection [12] $\widetilde{\nabla}$ with respect to the reductive decomposition $\mathfrak{g}=\mathfrak{m} \oplus \mathfrak{k}$ is determined by its value at $x$

$$
\left(\widetilde{\nabla}_{X} Y\right)_{x}=\mu\left(\left[\mu^{-1}(X), \mu^{-1}(Y)\right]_{\mathfrak{m}}\right), \quad X, Y \in T_{x} M .
$$

The tensor field $S=\nabla-\widetilde{\nabla}$ is the homogeneous Riemannian structure associated to the reductive decomposition $\mathfrak{g}=\mathfrak{m} \oplus \mathfrak{k}$.

Ambrose-Singer Theorem states that a connected, simply connected and complete Riemannian manifold is homogeneous Riemannian if and only if it 
admits a homogeneous structure tensor. In the case that $(M, g)$ is just a connected Riemannian manifold, the existence of a homogeneous structure tensor implies that $(M, g)$ is locally homogeneous. Tricerri and Vanhecke [18] gave a classification of the homogeneous Riemannian structure tensors in eight invariant classes: the class $\{S=0\}$ of symmetric structures, the total space denoted by $\mathcal{S}$, three irreducible classes under the action of the group $O(n)$

$$
\begin{aligned}
& \mathcal{S}_{1}=\left\{S \in \mathcal{S} / S_{X Y Z}=g(X, Y) \varphi(Z)-g(X, Z) \varphi(Y), \varphi \in \Gamma\left(T^{*} M\right)\right\} \\
& \mathcal{S}_{2}=\left\{S \in \mathcal{S} / \mathfrak{S}_{X Y Z} S_{X Y Z}=0, \quad c_{12}(S)=0\right\} \\
& \mathcal{S}_{3}=\left\{S \in \mathcal{S} / S_{X Y Z}+S_{Y X Z}=0\right\}
\end{aligned}
$$

and their direct sums

$$
\begin{aligned}
& \mathcal{S}_{1} \oplus \mathcal{S}_{2}=\left\{S \in \mathcal{S} / \mathbb{S}_{X Y Z} S_{X Y Z}=0\right\} \\
& \mathcal{S}_{1} \oplus \mathcal{S}_{3}=\left\{S \in \mathcal{S} / S_{X Y Z}+S_{Y X Z}=2 g(X, Y) \varphi(Z)-g(X, Z) \varphi(Y)\right. \\
& \mathcal{S}_{2} \oplus \mathcal{S}_{3}=\left\{S \in \mathcal{S} / c_{12}(S)=0\right\}
\end{aligned}
$$

where $c_{12}(S)_{p}(Z)=\sum_{i} S_{e_{i} e_{i} Z}$ for any orthonormal base $\left\{e_{i}\right\}_{i=1, \ldots, n}$ of $T_{p} M$.

\subsection{The reduced metric in a principal bundle}

Let $\pi: \bar{M} \rightarrow M$ be an $H$-principal bundle, where $\bar{M}$ is a Riemannian manifold with metric $\bar{g}$ and $H$ acts on $\bar{M}$ by isometries. Although it is not essential, the action of isometries are understood as left and hence $\pi$ is a left principal bundle. Let $\bar{x} \in \bar{M}$ and let $V_{\bar{x}} \bar{M}$ denote the vertical subspace at $\bar{x}$. If we take the orthogonal complement $H_{\bar{x}} \bar{M}=\left(V_{\bar{x}} \bar{M}\right)^{\perp}$ of $V_{\bar{x}} \bar{M}$ in $T_{\bar{x}} \bar{M}$ with respect to the metric $\bar{g}$ we have

$$
T_{\bar{x}} \bar{M}=V_{\bar{x}} \bar{M} \oplus H_{\bar{x}} \bar{M}
$$

Morever, as $H$ acts by isometries, the horizontal subspaces $H_{\bar{x}} \bar{M}$ are preserved by the action of $H$, and the decomposition (3) leads to the so called mechanical connection in the principal bundle $\bar{M} \rightarrow M$. In this situation there is a unique Riemannian metric $g$ in $M$ such that the restriction $\pi_{*}: H_{\bar{x}} \bar{M} \rightarrow T_{\pi(\bar{x})} M$ is an isometry at every $\bar{x} \in \bar{M}$. Obviously, the metric $g$ satisfies

$$
g(X, Y) \circ \pi=\bar{g}\left(X^{H}, Y^{H}\right) \quad \forall X, Y \in \mathfrak{X}(M)
$$

where $X^{H}$ and $Y^{H}$ denote the horizontal lift of $X$ and $Y$ with respect to the mechanical connection. To complete the notation, in the following, for a vector $Z \in T_{\bar{x}} \bar{M}$, we will denote by $Z^{h} \in H_{\bar{x}} \bar{M}$ the horizontal part of $Z$ with respect to the mechanical connection. In particular,

$$
Z^{h}=\left(\pi_{*}(Z)\right)^{H}
$$


Proposition 2.1 In the situation above, if $\bar{\nabla}$ is the Levi-Civita connection for the metric $\bar{g}$, then the Levi-Civita connection $\nabla$ for the reduced metric $g$ is given by

$$
\nabla_{X} Y=\pi_{*}\left(\bar{\nabla}_{X^{H}} Y^{H}\right), \quad \forall X, Y \in \mathfrak{X}(M) .
$$

Proof. Since the structure group $H$ acts by isometries, it also acts by affine transformations of $\bar{\nabla}$. Thus the vector field $\bar{\nabla}_{X^{H}} Y^{H}$ is projectable and the operator $D_{X} Y=\pi_{*}\left(\bar{\nabla}_{X^{H}} Y^{H}\right)$ is well defined. It is a direct computation to show that $D$ fulfills the properties of a linear connection in $M$. For $X, Y, Z \in \mathfrak{X}(M)$, from (4) and (5) we have

$$
\begin{aligned}
g\left(D_{X} Y, Z\right) \circ \pi+g\left(Y, D_{X}, Z\right) \circ \pi & =\bar{g}\left(\left(\bar{\nabla}_{X^{H}} Y^{H}\right)^{h}, Z^{H}\right)+\bar{g}\left(Y^{H},\left(\bar{\nabla}_{X^{H}} Z^{H}\right)^{h}\right) \\
& =\bar{g}\left(\bar{\nabla}_{X^{H}} Y^{H}, Z^{H}\right)+\bar{g}\left(Y^{H}, \bar{\nabla}_{X^{H}} Z^{H}\right) \\
& =X^{H}\left(\bar{g}\left(Y^{H}, Z^{H}\right)\right) .
\end{aligned}
$$

Hence $g\left(D_{X} Y, Z\right)+g\left(Y, D_{X} Z\right)=X(g(Y, Z))$ and the connection $D$ is metric. Finally, as $[X, Y]^{H}=\left[X^{H}, Y^{H}\right]^{h}$, the torsion tensor of $D$ is

$$
\begin{aligned}
T(X, Y) & =D_{X} Y-D_{Y} X-[X, Y] \\
& =\pi_{*}\left(\bar{\nabla}_{X^{H}} Y^{H}-\bar{\nabla}_{Y^{H}} X^{H}-\left[X^{H}, Y^{H}\right]\right) \\
& =0,
\end{aligned}
$$

and $D$ is the Levi-Civita connection for $g$.

\section{Main Results}

\subsection{Reduction by a normal subgroup of isometries}

Let $(\bar{M}, \bar{g})$ be a homogeneous Riemannian manifold. Let $\bar{G}$ be a group of isometries acting transitively on $\bar{M}$ and $H \triangleleft \bar{G}$ a normal subgroup acting freely on $\bar{M}$. The quotient $M=\bar{M} / H$ is thus endowed (cf. [13, Th. 9.16]) with a smooth structure such that $\pi: \bar{M} \rightarrow M$ is an $H$-principal bundle. By definition, the bundle $\pi: \bar{M} \rightarrow M$ is equipped with the mechanical connection and $M$ is Riemannian with the reduced metric $g$ as in (4). Since $H$ is normal, there is a well-defined action of the group $G=\bar{G} / H$ on $M$ given by

$$
\Phi: \begin{aligned}
G \times M & \rightarrow M \\
([\bar{a}],[\bar{x}]) & \mapsto \Phi_{[\bar{a}]}([\bar{x}])=\left[\Phi_{\bar{a}}(\bar{x})\right]
\end{aligned}
$$

where $[\bar{a}]$ and $[\bar{x}]$ denotes the classes modulo $H$ of $\bar{a} \in \bar{G}$ and $\bar{x} \in \bar{M}$ respectively, and $\Phi_{\bar{a}}$ denotes the action of $\bar{G}$ on $\bar{M}$. The action of $G$ is obviously transitive but needs not be effective. If it is not, we replace $G$ by $G / N$, where $N$ is the kernel of the map $G \rightarrow \operatorname{Isom}(M), a \mapsto \Phi_{a}, a \in G$.

Proposition 3.1 The group $G$ acts on $(M, g)$ by isometries. 
Proof. The action (7) can be written as $\pi \circ \Phi_{\bar{a}}=\Phi_{a} \circ \pi$, for $a=[\bar{a}]$. This implies that $\bar{G}$ preserves vertical subspaces and, acting by isometries, also their horizontal complements. Hence, the horizontal lift of $\left(\Phi_{a}\right)_{*}(X)$ is $\left(\Phi_{\bar{a}}\right)_{*}\left(X^{H}\right)$ for all $X \in \mathfrak{X}(M)$. In addition, for $X, Y \in \mathfrak{X}(M)$

$$
\begin{aligned}
g\left(\left(\Phi_{a}\right)_{*}(X),\left(\Phi_{a}\right)_{*}(Y)\right) \circ \pi & =\bar{g}\left(\left(\Phi_{a}\right)_{*}(X)^{H},\left(\Phi_{a}\right)_{*}(Y)^{H}\right) \\
& =\bar{g}\left(\left(\Phi_{\bar{a}}\right)_{*}\left(X^{H}\right),\left(\Phi_{\bar{a}}\right)_{*}\left(Y^{H}\right)\right) \\
& =\bar{g}\left(X^{H}, Y^{H}\right) \\
& =g(X, Y) \circ \pi
\end{aligned}
$$

and then $\Phi_{a}$ is an isometry.

From this last Proposition, the manifold $(M, g)$ is homogeneous Riemannian. We will call it the reduced homogeneous Riemannian manifold.

Remark 3.2 Note that Proposition 3.1 shows that the horizontal distribution is invariant by $\bar{G}$. This means that the mechanical connection is $\bar{G}$-invariant, an important fact that will be used in 33.3 .

Let $\bar{x} \in \bar{M}$ and $x=\pi(\bar{x}) \in M$. We denote by $\bar{K}$ the isotropy group of $\bar{x}$ under the action of $\bar{G}$, and by $K$ the corresponding isotropy group of $x$ under the action of $G$. We also denote their Lie algebras by $\overline{\mathfrak{k}}$ and $\mathfrak{k}$ respectively. Then we have

Lemma 3.3 Let $\tau: \bar{G} \rightarrow G$ be the quotient homomorphism. Then $K=\tau(\bar{K})$ and the restriction $\left.\tau\right|_{\bar{K}}: \bar{K} \rightarrow K$ is an isomorphism of groups.

Proof. It is obvious from (77) that $\tau(\bar{K}) \subset K$. Let now $k \in K$ and take $\bar{a} \in \bar{G}$ such that $k=\tau(\bar{a})$. Then for any $x \in M$, we have $x=\Phi_{k}(x)=\pi\left(\Phi_{\bar{a}}(\bar{x})\right)$, and then $\Phi_{\bar{a}}(\bar{x})$ is in the same fibre as $\bar{x}$. Hence there exists $h \in H$ such that $\Phi_{h} \circ \Phi_{\bar{a}}(\bar{x})=\bar{x}$, so $h \bar{a} \in \bar{K}$. Since $\tau(h \bar{a})=\tau(\bar{a})=k$ we have $k \in \tau(\bar{K})$. For the injectivity of $\left.\tau\right|_{\bar{K}}$, let $\bar{k}_{1}, \bar{k}_{2} \in \bar{K}$ such that $\tau\left(\bar{k}_{1}\right)=\tau\left(\bar{k}_{2}\right)$. There exists $h \in H$ such that $h \bar{k}_{1}=\bar{k}_{2}$. Then $\bar{k}_{1}^{-1} h \bar{k}_{1}=\bar{k}_{1}^{-1} \bar{k}_{2}$, so $\bar{k}_{1}^{-1} \bar{k}_{2} \in \bar{K} \cap H$. But since $H$ acts freely, $\bar{k}_{1}^{-1} \bar{k}_{2}=\bar{e}$, and then $\bar{k}_{1}=\bar{k}_{2}$.

Theorem 3.4 Let $(\bar{M}, \bar{g})$ be a connected homogeneous Riemannian manifold and let $\bar{G}$ be a group of isometries acting transitively and effectively in $\bar{M}$. Let $H \triangleleft \bar{G}$ be a normal subgroup acting freely in $\bar{M}$. Then every homogeneous structure tensor $\bar{S}$ associated to $\bar{G}$ induces a homogeneous structure tensor $S$ associated to $G=\bar{G} / H$ in the reduced Riemannian manifold $M=\bar{M} / H$.

Proof. Let $\bar{x} \in \bar{M}$ and $x=\pi(\bar{x}) \in M$, and let $\overline{\mathfrak{g}}$ be the Lie algebra of $\bar{G}$. For any reductive decomposition $\overline{\mathfrak{g}}=\overline{\mathfrak{m}} \oplus \overline{\mathfrak{k}}$ associated to $\bar{S}$, the restriction isomorphism $\bar{\mu}: \overline{\mathfrak{m}} \rightarrow T_{\bar{x}} \bar{M}$ induces from $\bar{g}$ a positive definite bilinear form $B$ in $\overline{\mathfrak{m}}$. Moreover, by the commutativity of (2) the bilinear form $B$ is $A d(\bar{K})$-invariant, that is,

$$
B(A d(\bar{k}) \xi, A d(\bar{k}) \eta)=B(\xi, \eta) \quad \forall \bar{k} \in \bar{K} .
$$


Then (3) induces an orthogonal and $A d(\bar{K})$-invariant decomposition

$$
\overline{\mathfrak{m}}=\overline{\mathfrak{m}}^{v} \oplus \overline{\mathfrak{m}}^{h}
$$

i.e., $\operatorname{Ad}(\bar{K})\left(\overline{\mathfrak{m}}^{v}\right) \subset \overline{\mathfrak{m}}^{v}$ and $\operatorname{Ad}(\bar{K})\left(\overline{\mathfrak{m}}^{h}\right) \subset \overline{\mathfrak{m}}^{h}$.

Let $\mathfrak{g}=\overline{\mathfrak{g}} / \mathfrak{h}$ be the lie algebra of $G$ and $\mu: \mathfrak{g} \rightarrow T_{x} M$ the corresponding infinitesimal action at $x$. For any $\bar{\xi} \in \overline{\mathfrak{g}}$, by (7) we have

$$
\begin{aligned}
\pi_{*} \circ \bar{\mu}(\bar{\xi}) & =\pi_{*}\left(\left.\frac{d}{d t}\right|_{t=0} \Phi_{\exp (t \bar{\xi})}(\bar{x})\right) \\
& =\left.\frac{d}{d t}\right|_{t=0}\left(\pi \circ \Phi_{\exp (t \bar{\xi})}\right)(\bar{x}) \\
& =\left.\frac{d}{d t}\right|_{t=0} \Phi_{\tau(\exp (t \bar{\xi}))}(\pi(\bar{x})) \\
& =\left.\frac{d}{d t}\right|_{t=0} \Phi_{\exp \left(t \tau_{*}(\bar{\xi})\right)}(x) \\
& =\mu \circ \tau_{*}(\bar{\xi})
\end{aligned}
$$

which means that the following diagram is commutative

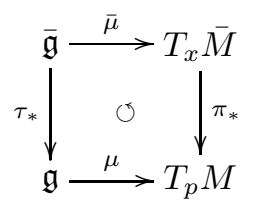

Restrictions to $\overline{\mathfrak{m}}^{h}$ and $\overline{\mathfrak{m}}^{v}$ give commutative diagrams

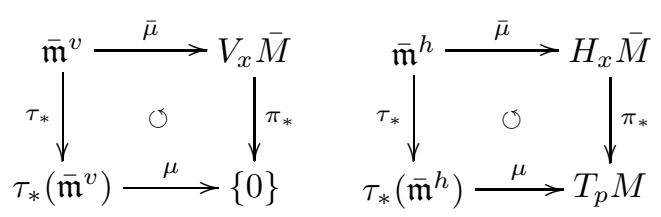

which shows that $\tau_{*}: \mathfrak{m}^{h} \rightarrow \tau_{*}\left(\mathfrak{m}^{h}\right)$ and $\mu: \tau_{*}\left(\mathfrak{m}^{h}\right) \rightarrow T_{x} M$ are isomorphisms, and $\tau_{*}\left(\mathfrak{m}^{v}\right) \subset \mathfrak{k}$. In addition, by Lemma 3.3 the restriction of $\tau_{*}: \overline{\mathfrak{g}} \rightarrow \mathfrak{g}$ to $\overline{\mathfrak{k}}$ is an isomorphism of Lie algebras from $\overline{\mathfrak{k}}$ to $\mathfrak{k}$. Therefore, denoting by $\mathfrak{m}$ the image $\tau_{*}\left(\overline{\mathfrak{m}}^{h}\right)$, we have the decomposition

$$
\mathfrak{g}=\mathfrak{m} \oplus \mathfrak{k}
$$


Let $k \in K$ and $\xi \in \mathfrak{m}$, and let $\bar{k} \in \bar{K}$ and $\bar{\xi} \in \overline{\mathfrak{m}}^{h}$ be such that $\tau(\bar{k})=k$ and $\tau_{*}(\bar{\xi})=\xi$ we have

$$
\begin{aligned}
\operatorname{Ad}(k)(\xi) & =\operatorname{Ad}(\tau(\bar{k}))\left(\tau_{*}(\bar{\xi})\right) \\
& =\mu^{-1} \circ \Phi_{\tau(\bar{k})} \circ \mu\left(\tau_{*}(\bar{\xi})\right) \\
& =\mu^{-1} \circ \Phi_{\tau(\bar{k})} \circ \pi_{*}(\bar{\mu}(\bar{\xi})) \\
& =\mu^{-1} \circ \pi_{*} \circ \Phi_{\bar{k}}(\bar{\mu}(\bar{\xi})) \\
& =\mu^{-1} \circ \pi_{*} \circ \bar{\mu}(\operatorname{Ad}(\bar{k})(\bar{\xi})) \\
& =\mu^{-1} \circ \mu \circ \tau_{*}(\operatorname{Ad}(\bar{k})(\bar{\xi})) \\
& =\tau_{*}(\operatorname{Ad}(\bar{k})(\bar{\xi})) .
\end{aligned}
$$

Since $\overline{\mathfrak{m}}^{h}$ is $A d(\bar{K})$-invariant we deduce that $A d(k)(\mathfrak{m}) \subset \tau_{*}\left(\overline{\mathfrak{m}}^{h}\right)=\mathfrak{m}$, which proves that (10) is a reductive decomposition.

The homogeneous structure tensor associated to (10) at $x$ is given by [18, p.24]

$$
\left(S_{x}\right)_{X} Y=\left(\nabla_{Y} \xi^{*}\right)_{x} \quad X, Y \in T_{x} M
$$

where $\xi^{*}$ is the vector field given by the infinitesimal action of $\xi \in \mathfrak{m}$ with $\xi_{x}^{*}=\mu(\xi)=X$. Let $\bar{\xi} \in \overline{\mathfrak{m}}^{h}$ be such that $\tau_{*}(\bar{\xi})=\xi$ then

$$
\begin{aligned}
\left(S_{x}\right)_{X} Y=\left(\nabla_{Y} \xi^{*}\right)_{x} & =\pi_{*}\left(\left(\bar{\nabla}_{Y^{H}}\left(\xi^{*}\right)^{H}\right)_{\bar{x}}\right) \\
& =\pi_{*}\left(\left(\bar{\nabla}_{Y^{H}} \bar{\xi}^{*}\right)\right)-\pi_{*}\left(\left(\bar{\nabla}_{Y^{H}}\left(\bar{\xi}^{*}\right)^{v}\right)_{\bar{x}}\right) .
\end{aligned}
$$

Now let $\bar{Z} \in T_{\bar{x}} \bar{M}$ be an horizontal vector, since $\bar{\xi}_{\bar{x}}^{*}$ is horizontal

$$
\bar{g}\left(\left(\bar{\nabla}_{Y^{H}}\left(\bar{\xi}^{*}\right)^{v}\right)_{\bar{x}}, \bar{Z}\right)=Y^{H} \bar{g}\left(\left(\bar{\xi}^{*}\right)^{v}, \bar{Z}\right)-\bar{g}\left(\left(\bar{\xi}^{*}\right)_{\bar{x}}^{v}, \bar{\nabla}_{Y^{H}} \bar{Z}\right)=0 .
$$

Hence by [18, p.24] and (9)

$$
\left(S_{x}\right)_{X} Y=\pi_{*}\left(\left(\bar{S}_{\bar{x}}\right)_{X^{H}} Y^{H}\right) \quad X, Y \in T_{x} M .
$$

Finally we extend $S_{x}$ to the whole $M$ with the action of $G$ to obtain a homogeneous structure tensor $S$.

We shall call the tensor field $S$ the reduced homogeneous structure tensor.

Corollary 3.5 The reduced homogeneous structure can be expressed as

$$
S_{X} Y=\pi_{*}\left(\bar{S}_{X^{H}} Y^{H}\right) \quad X, Y \in \mathfrak{X}(M) .
$$

Proof. Let $\bar{a} \in \bar{G}$ and $a=\tau(\bar{a}) \in G$ we proved that the horizontal lift of $\left(\Phi_{a}\right)_{*}(X)$ is $\left(\Phi_{\bar{a}}\right)_{*}\left(X^{H}\right)$ for all $X \in \mathfrak{X}(M)$. This together with the invariance of $\bar{S}$ by $\bar{G}$ and the invariance of $S$ by $G$ gives (12). 


\subsection{The space of tensors reducing to a given tensor}

Suppose we are now in the situation of Theorem 3.4 and we have a homogeneous structure tensor $S$ associated to $G$ in the reduced manifold $M$. Using diagram (8) we can define the subspaces of $\overline{\mathfrak{g}}$

$$
\overline{\mathfrak{m}}^{h}=\tau_{*}^{-1}(\mathfrak{m}) \cap \bar{\mu}^{-1}\left(H_{\bar{x}} \bar{M}\right) \quad \text { and } \quad \overline{\mathfrak{m}}^{v}=\mathfrak{h} .
$$

Then the decomposition

$$
\overline{\mathfrak{g}}=\overline{\mathfrak{m}} \oplus \overline{\mathfrak{k}}, \quad \text { with } \quad \overline{\mathfrak{m}}=\overline{\mathfrak{m}}^{v} \oplus \overline{\mathfrak{m}}^{h}
$$

is a reductive decomposition. Indeed, since $H$ is normal in $\bar{G}$ it is obvious that $A d(\bar{K})(\mathfrak{h}) \subset \mathfrak{h}$. On the other hand, for $\bar{k} \in \bar{K}$ and $\bar{\xi} \in \overline{\mathfrak{m}}^{h}$, as $\bar{\mu}(\operatorname{Ad}(\bar{k})(\bar{\xi}))=$ $\left(\Phi_{\bar{k}}\right)_{*}(\bar{\mu}(\bar{\xi}))$, we have $\bar{\mu}(A d(\bar{k})(\bar{\xi})) \in H_{\bar{x}} \bar{M}$ and $\tau_{*}(A d(\bar{k})(\bar{\xi})) \in \mathfrak{m}$, and then $\operatorname{Ad}(\bar{k})(\bar{\xi}) \in \overline{\mathfrak{m}}^{h}$. The homogeneous structure tensor associated to this decomposition at $\bar{x}$ is (see, for example [10])

$$
\left(\bar{S}_{\bar{x}}\right)_{\bar{X} \bar{Y} \bar{Z}}=\frac{1}{2}\left(B\left([\bar{\xi}, \bar{\eta}]_{\overline{\mathfrak{m}}}, \bar{\zeta}\right)-B\left([\bar{\eta}, \bar{\zeta}]_{\overline{\mathfrak{m}}}, \bar{\xi}\right)+B\left([\bar{\zeta}, \bar{\xi}]_{\overline{\mathfrak{m}}}, \bar{\eta}\right)\right), \quad \bar{X}, \bar{Y}, \bar{Z} \in T_{\bar{x}} \bar{M}
$$

where $\bar{\xi}, \bar{\eta}, \bar{\zeta} \in \overline{\mathfrak{m}}$ are such that their images by $\bar{\mu}$ are $X, Y, Z$, and $B$ is the bilinear form induced on $\overline{\mathfrak{m}}$ from $T_{\bar{x}} \bar{M}$ by $\bar{\mu}$. Note that we have exactly the situation in the proof of Theorem 3.4 , so the homogeneous structure tensor $\bar{S}$ associated to (13) reduces to $S$.

We can construct all other homogeneous structures in $\bar{M}$ associated to $\bar{G}$ by changing $\overline{\mathfrak{m}}$ in (13) by the graph

$$
\overline{\mathfrak{m}}^{\varphi}=\{X+\varphi(X) / X \in \overline{\mathfrak{m}}\}
$$

of an $A d(\bar{K})$-equivariant map $\varphi: \mathfrak{h} \oplus \overline{\mathfrak{m}}^{h} \rightarrow \overline{\mathfrak{k}}$. The condition that the new homogeneous structure tensors reduce to $S$ is equivalent to the condition $\varphi_{\mid \overline{\mathfrak{m}}^{h}}=0$. So the family of homogeneous structure tensors that reduce to $S$ is parameterized by the set of $A d(\bar{K})$-equivariant maps $\varphi: \mathfrak{h} \rightarrow \overline{\mathfrak{k}}$. For the sake of convenience we will denote by the same $\varphi$ both $\varphi: \mathfrak{h} \rightarrow \overline{\mathfrak{k}}$ and its extension by zero to $\overline{\mathfrak{m}}=\mathfrak{h} \oplus \overline{\mathfrak{m}}^{h}$. The expression of the homogeneous structure tensor $\bar{S}^{\varphi}$ associated to this map is the same as in (14) by changing $\overline{\mathfrak{m}}$ to $\overline{\mathfrak{m}}^{\varphi}, B$ to the induced bilinear form $B^{\varphi}$ in $\overline{\mathfrak{m}}^{\varphi}$ and the $\bar{\xi}, \bar{\eta}, \bar{\zeta}$ to $\bar{\xi}^{\prime}=\bar{\xi}+\varphi(\bar{\xi}), \bar{\eta}^{\prime}=\bar{\eta}+\varphi(\bar{\eta})$, $\bar{\zeta}^{\prime}=\bar{\zeta}+\varphi(\bar{\zeta}) \in \overline{\mathfrak{m}}^{\varphi}$. As

$$
\left[\bar{\xi}^{\prime}, \bar{\eta}^{\prime}\right]_{\overline{\mathfrak{m}} \varphi}=[\bar{\xi}, \bar{\eta}]_{\overline{\mathfrak{m}} \varphi}+[\bar{\xi}, \varphi(\bar{\eta})]_{\overline{\mathfrak{m}} \varphi}+[\varphi(\bar{\xi}), \bar{\eta}]_{\overline{\mathfrak{m}} \varphi}+[\varphi(\bar{\xi}), \varphi(\bar{\eta})]_{\overline{\mathfrak{m}} \varphi}
$$

and $[\varphi(\bar{\xi}), \varphi(\bar{\eta})]_{\overline{\mathfrak{m}} \varphi}=0$ we have that

$$
\begin{aligned}
B^{\varphi}\left(\left[\bar{\xi}^{\prime}, \bar{\eta}^{\prime}\right]_{\overline{\mathfrak{m}} \varphi}, \bar{\zeta}^{\prime}\right) & =B^{\varphi}\left([\bar{\xi}, \bar{\eta}]_{\overline{\mathfrak{m}} \varphi}, \bar{\zeta}^{\prime}\right)+B^{\varphi}\left([\bar{\xi}, \varphi(\bar{\eta})]_{\overline{\mathfrak{m}} \varphi}+[\varphi(\bar{\xi}), \bar{\eta}]_{\overline{\mathfrak{m}} \varphi}, \bar{\zeta}^{\prime}\right) \\
& =B\left([\bar{\xi}, \bar{\eta}]_{\overline{\mathfrak{m}}}, \bar{\zeta}\right)+B([\bar{\xi}, \varphi(\bar{\eta})]+[\varphi(\bar{\xi}), \bar{\eta}], \bar{\zeta}),
\end{aligned}
$$

where one has to take into account that the isomorphism $\overline{\mathfrak{m}} \rightarrow \overline{\mathfrak{m}}^{\varphi}, \bar{\xi} \mapsto \bar{\xi}+\varphi(\bar{\xi})$ is an isometry with respect to $B$ and $B^{\varphi}$. Hence

$$
\begin{aligned}
\left(\bar{S}_{\bar{x}}^{\varphi}\right)_{\bar{X} \bar{Y} \bar{Z}} & =\left(\bar{S}_{\bar{x}}\right)_{\bar{X} \bar{Y} \bar{Z}}+\frac{1}{2}\{B([\bar{\xi}, \varphi(\bar{\eta})]+[\varphi(\bar{\xi}), \bar{\eta}], \bar{\zeta}) \\
& -B([\bar{\eta}, \varphi(\bar{\zeta})]+[\varphi(\bar{\eta}), \bar{\zeta}], \bar{\xi})+B([\bar{\zeta}, \varphi(\bar{\xi})]+[\varphi(\bar{\zeta}), \bar{\xi}], \bar{\eta})\} \cdot(15)
\end{aligned}
$$


The summands involving $B$ define a tensor field $P^{\varphi}$ globally defined in $\bar{M}$ by the left action of $\bar{G}$. More precisely, for any $\bar{y} \in \bar{M}$, with $\bar{y}=\Phi_{\bar{a}}(\bar{x}), \bar{a} \in \bar{G}$, this tensor is

$$
\begin{aligned}
\left(P_{\bar{y}}^{\varphi}\right)_{\bar{X} \bar{Y} \bar{Z}} & =\frac{1}{2}\left\{B_{\bar{y}}\left(\left[\bar{\xi}, \varphi_{\bar{y}}(\bar{\eta})\right]+\left[\varphi_{\bar{y}}(\bar{\xi}), \bar{\eta}\right], \bar{\zeta}\right)-B_{\bar{y}}\left(\left[\bar{\eta}, \varphi_{\bar{y}}(\bar{\zeta})\right]+\left[\varphi_{\bar{y}}(\bar{\eta}), \bar{\zeta}\right], \bar{\xi}\right)\right. \\
& \left.+B_{\bar{y}}\left(\left[\bar{\zeta}, \varphi_{\bar{y}}(\bar{\xi})\right]+\left[\varphi_{\bar{y}}(\bar{\zeta}), \bar{\xi}\right], \bar{\eta}\right)\right\}
\end{aligned}
$$

for $\bar{X}, \bar{Y}, \bar{Z} \in T_{\bar{y}} \bar{M}$, where

$$
\begin{aligned}
\overline{\mathfrak{m}}_{\bar{y}} & :=\operatorname{Ad}(\bar{a})(\overline{\mathfrak{m}}), \quad \overline{\mathfrak{k}}_{\bar{y}}:=\operatorname{Ad}(\bar{a})(\overline{\mathfrak{k}}), \\
\varphi_{\bar{y}} & :=\operatorname{Ad}(\bar{a}) \circ \varphi \circ \operatorname{Ad}\left(\bar{a}^{-1}\right): \mathfrak{h} \rightarrow \overline{\mathfrak{k}}_{\bar{y}},
\end{aligned}
$$

$B_{\bar{y}}$ is the bilinear form on $\overline{\mathfrak{m}}_{\bar{y}}$ induced from $\bar{g}_{\bar{y}}$ by

$$
\bar{\mu}_{\bar{y}}:=\left(\Phi_{\bar{a}}\right)_{*} \circ \bar{\mu} \circ \operatorname{Ad}\left(\bar{a}^{-1}\right): \overline{\mathfrak{m}}_{\bar{y}} \rightarrow T_{\bar{y}} \bar{M},
$$

and $\bar{\xi}, \bar{\eta}, \bar{\zeta} \in \overline{\mathfrak{m}}_{\bar{y}}$ are such that their images by $\bar{\mu}_{\bar{y}}$ are $\bar{X}, \bar{Y}, \bar{Z}$ respectively.

We have then proved

Proposition 3.6 In the situation of Theorem 3.4. let $S$ be a homogeneous structure tensor in $M$ associated to $G$. Then the space of homogeneous structure tensors in $\bar{M}$ associated to $\bar{G}$ and reducing to $S$ is a vector space isomorphic to the space of $A d(\bar{K})$-equivariant maps $\varphi: \mathfrak{h} \rightarrow \overline{\mathfrak{k}}$. Moreover, the isomorphism is given by

$$
\varphi \mapsto \bar{S}^{\varphi}=\bar{S}+P^{\varphi}
$$

where $\bar{S}$ is the homogeneous structure associated to the decomposition (13) and $P^{\varphi}$ is given in (16).

\subsection{Reduction in a principal bundle}

We have noted in Remark 3.2 that the normality of the group $H$ gives the invariance of the mechanical connection. This implies that the connection form $\omega$ is $A d(\bar{G})$-equivariant, i.e.,

$$
\Phi_{\bar{a}}^{*} \omega=A d(\bar{a}) \cdot \omega, \quad \forall \bar{a} \in \bar{G},
$$

where $A d(\bar{a}) \cdot \omega$ denotes the 1 -form in $\bar{M}$ with values in $\mathfrak{h}$ given by

$$
(\operatorname{Ad}(\bar{a}) \cdot \omega)(\bar{X})=\operatorname{Ad}(\bar{a})(\omega(\bar{X})) .
$$

The canonical linear connection $\tilde{\bar{\nabla}}=\bar{\nabla}-\bar{S}$ of the reductive decomposition $\overline{\mathfrak{g}}=\overline{\mathfrak{m}} \oplus \overline{\mathfrak{k}}$ at $\bar{x}$ is characterized by the following property: for every $\bar{\xi} \in \overline{\mathfrak{m}}$, the parallel displacement with respect to $\widetilde{\nabla}$ along the curve $\gamma(t)=\Phi_{\exp (t \bar{\xi})}(\bar{x})$, 
from $\bar{x}$ to $\gamma(t)$, is equal to $\left(\Phi_{\exp (t \bar{\xi})}\right)_{*}$ (see [12, Vol. II, Ch. X, Corollary 2.5 ]). Hence, infinitesimally we have that

$$
\left(\widetilde{\bar{\nabla}}_{\bar{X}} \omega\right)_{\bar{x}}=\operatorname{ad}\left(\bar{\mu}^{-1}(\bar{X})\right) \cdot \omega_{\bar{x}}, \quad \forall \bar{X} \in T_{\bar{x}} \bar{M},
$$

and by the invariance of $\widetilde{\bar{\nabla}}$ by $\bar{G}$

$$
\left(\widetilde{\bar{\nabla}}_{\bar{X}} \omega\right)_{\bar{y}}=\operatorname{ad}\left(\bar{\mu}_{\bar{y}}^{-1}(\bar{X})\right) \cdot \omega_{\bar{y}}, \quad \forall \bar{y} \in \bar{M}, \forall \bar{X} \in T_{\bar{y}} \bar{M},
$$

that is, the covariant derivative of $\omega$ by the connection $\widetilde{\widetilde{\nabla}}$ is proportional to itself by a suitable linear operator. We note that, in particular, if $H$ is contained in the center of $\bar{G}$, the linear operator is null, hence $\omega$ is invariant by $\bar{G}$. If $H$ is just a normal subgroup not contained in the center, condition (18) comes from the equivariance of $\omega$.

The preceding discussion suggests to study the reduction of homogeneous structure tensors $\bar{S}$ in a principal bundle without the use of the group $\bar{G}$. More precisely, in Theorem 3.4 the group $\bar{G}$ (and its reductive decomposition) associated to the tensor $\bar{S}$ was a key ingredient. We now begin with any tensor $\bar{S}$ in a manifold $(\bar{M}, \bar{g})$ where a group $H$ acts by isometries (and such that $\bar{M} \rightarrow \bar{M} / H=M$ is a principal bundle) satisfying Ambrose-Singer equations and an additional algebraic condition for the mechanical connection analogous to (18). Then the tensor $\bar{S}$ can also be projected without using any reductive decomposition as we can see in the following result.

Theorem 3.7 Let $(\bar{M}, \bar{g})$ be a Riemannian manifold. Let $\pi: \bar{M} \rightarrow M$ be a principal bundle with structure group $H$ acting on $\bar{M}$ by isometries, and endowed with the mechanical connection $\omega$. For every $H$-invariant homogeneous Riemannian structure tensor $\bar{S}$ with canonical linear connection $\widetilde{\bar{\nabla}}$, if

$$
\widetilde{\bar{\nabla}} \omega=\alpha \cdot \omega
$$

for certain 1-form $\alpha$ in $\bar{M}$ taking values in $\operatorname{End}(\mathfrak{h})$, then the tensor field $S$ defined by

$$
S_{X} Y=\pi_{*}\left(\bar{S}_{X^{H}} Y^{H}\right) \quad X, Y \in \mathfrak{X}(M)
$$

is a homogeneous Riemannian structure tensor in $(M, g)$, where $g$ is the reduced Riemannian metric.

Proof. First note that $H$-invariance of $\bar{S}$ implies that $\bar{S}_{X^{H}} Y^{H}$ is projectable and then $S$ is well defined. Since the structure group $H$ acts by isometries, the Levi-Civita connection $\bar{\nabla}$ of $\bar{g}$ is $H$-invariant, which implies that $\widetilde{\bar{\nabla}}=\bar{\nabla}-\bar{S}$ is also $H$-invariant. Now from condition (19) we have that for all $X, Y \in \mathfrak{X}(M)$

$$
\omega\left(\widetilde{\bar{\nabla}}_{X^{H}} Y^{H}\right)=X^{H}\left(\omega\left(Y^{H}\right)\right)-\left(\widetilde{\bar{\nabla}}_{X^{H}} \omega\right)\left(Y^{H}\right)=-\alpha\left(X^{H}\right) \cdot \omega\left(Y^{H}\right)=0,
$$


so that $\widetilde{\bar{\nabla}}_{X^{H}} Y^{H}$ is horizontal. If we define $\widetilde{\nabla}=\nabla-S, \nabla$ being the Levi-Civita connection of $g$, then $\widetilde{\bar{\nabla}}_{X^{H}} Y^{H}$ projects to $\widetilde{\nabla}_{X^{H}} Y^{H}$. Hence by $H$-invariance,

$$
\left(\widetilde{\nabla}_{X} Y\right)^{H}=\widetilde{\bar{\nabla}}_{X^{H}} Y^{H}
$$

We now prove that $S$ satisfies Ambrose-Singer equations (equivalent to those in (11)):

$$
\widetilde{\nabla} g=0, \quad \widetilde{\nabla} \widetilde{R}=0, \quad \widetilde{\nabla} S=0,
$$

where $\widetilde{R}$ is the curvature tensor of $\widetilde{\nabla}$ and $\widetilde{R}$ and $S$ are seen as $(0,4)$ and $(0,3)$ tensors respectively by lowering their contravariant index with respect to $g$.

For the first equation, taking into account (21), we have for $U, X, Y \in \mathfrak{X}(M)$

$$
\begin{aligned}
\left(\widetilde{\nabla}_{U} g\right)(X, Y) \circ \pi & =U(g(X, Y)) \circ \pi-g\left(\widetilde{\nabla}_{U} X, Y\right) \circ \pi-g\left(X, \widetilde{\nabla}_{U} Y\right) \circ \pi \\
& =U^{H}\left(\bar{g}\left(X^{H}, Y^{H}\right)\right)-\bar{g}\left(\left(\widetilde{\nabla}_{U} X\right)^{H}, Y^{H}\right)-\bar{g}\left(X^{H},\left(\widetilde{\nabla}_{U} Y\right)^{H}\right) \\
& =U^{H}\left(\bar{g}\left(X^{H}, Y^{H}\right)\right)-\bar{g}\left(\widetilde{\bar{\nabla}}_{U^{H}} X^{H}, Y^{H}\right)-\bar{g}\left(X^{H}, \widetilde{\bar{\nabla}}_{U^{H}} Y^{H}\right) \\
& =\left(\widetilde{\bar{\nabla}}_{U^{H}} \bar{g}\right)\left(X^{H}, Y^{H}\right)
\end{aligned}
$$

and then since $\widetilde{\bar{\nabla}} \bar{g}=0$ we have $\widetilde{\nabla} g=0$.

For the third equation, let $U, X, Y, Z \in \mathfrak{X}(M)$. Then, again by (21), we have

$$
\begin{aligned}
\left(\widetilde{\nabla}_{U} S\right)_{X Y Z} \circ \pi= & U\left(S_{X Y Z}\right) \circ \pi-\left(S_{\widetilde{\nabla}_{U} X Y Z}\right) \circ \pi \\
& -\left(S_{X \widetilde{\nabla}_{U} Y}\right) \circ \pi-\left(S_{X Y \widetilde{\nabla}_{U} Z}\right) \circ \pi \\
= & U^{H}\left(\bar{S}_{X^{H} Y^{H} Z^{H}}\right)-\bar{S}_{\left(\widetilde{\nabla}_{U} X\right)^{H} Y^{H} Z^{H}} \\
& -\bar{S}_{X^{H}\left(\widetilde{\nabla}_{U} Y\right)^{H} Z^{H}}-\bar{S}_{X^{H} Y^{H}\left(\widetilde{\nabla}_{U} Z\right)^{H}} \\
= & U^{H}\left(\bar{S}_{X^{H} Y^{H} Z^{H}}\right)-\bar{S}_{\widetilde{\nabla}_{U^{H}} X^{H} Y^{H} Z^{H}} \\
& -\bar{S}_{X^{H} \tilde{\nabla}_{U^{H}} Y^{H} Z^{H}}-\bar{S}_{X^{H} Y^{H} \widetilde{\nabla}_{U} Z^{H}} \\
= & \left(\widetilde{\widetilde{\nabla}}_{U^{H}} \bar{S}\right)_{X^{H} Y^{H} Z^{H}}
\end{aligned}
$$

which vanishes as $\widetilde{\bar{\nabla}} \bar{S}=0$.

We now prove the second Ambrose-Singer equation. Let $\widetilde{\bar{R}}$ be the curvature tensor of $\widetilde{\bar{\nabla}}$. From equation (21), for $X, Y, Z \in \mathfrak{X}(M)$ we first have

$$
\begin{aligned}
\left(\widetilde{R}_{X Y} Z\right)^{H} & =\widetilde{\bar{\nabla}}_{X^{H}}\left(\widetilde{\nabla}_{Y} Z\right)^{H}-\widetilde{\bar{\nabla}}_{Y^{H}}\left(\widetilde{\nabla}_{X} Z\right)^{H}-\widetilde{\bar{\nabla}}_{[X, Y]^{H}} Z^{H} \\
& =\widetilde{\bar{\nabla}}_{X^{H}}\left(\widetilde{\bar{\nabla}}_{Y^{H}} Z^{H}\right)-\widetilde{\bar{\nabla}}_{Y^{H}}\left(\widetilde{\bar{\nabla}}_{X^{H}} Z^{H}\right)-\widetilde{\bar{\nabla}}_{\left[X^{H}, Y^{H}\right]^{h}} Z^{H} \\
& =\widetilde{\bar{\nabla}}_{X^{H}}\left(\widetilde{\bar{\nabla}}_{Y^{H}} Z^{H}\right)-\widetilde{\bar{\nabla}}_{Y^{H}}\left(\widetilde{\bar{\nabla}}_{X^{H}} Z^{H}\right)-\widetilde{\bar{\nabla}}_{\left[X^{H}, Y^{H}\right]} Z^{H}+\widetilde{\bar{\nabla}}_{\left[X^{H}, Y^{H}\right]^{v}} Z^{H} \\
& =\widetilde{\bar{R}}_{X^{H} Y^{H}} Z^{H}+\widetilde{\bar{\nabla}}_{\left[X^{H}, Y^{H}\right]^{v}} Z^{H} .
\end{aligned}
$$


We shall also denote by $\widetilde{\bar{R}}$ the $(0,4)$ tensor field associated to $\widetilde{\bar{R}}$ obtained by lowering the contravariant index with respect to $\bar{g}$. Then for $X, Y, Z, W \in \mathfrak{X}(M)$ one has

$$
\begin{aligned}
\widetilde{R}_{X Y Z W} \circ \pi & =\widetilde{\bar{R}}_{X^{H} Y^{H} Z^{H} W^{H}}+\bar{g}\left(\widetilde{\bar{\nabla}}_{\left[X^{H}, Y^{H}\right]^{v}} Z^{H}, W^{H}\right) \\
& =\widetilde{\bar{R}}_{X^{H} Y^{H} Z^{H} W^{H}}-\bar{g}\left(\widetilde{\bar{\nabla}}_{\Omega\left(X^{H}, Y^{H}\right)^{*}} Z^{H}, W^{H}\right),
\end{aligned}
$$

where $\Omega\left(X^{H}, Y^{H}\right)^{*}$ is the fundamental vector field associated to $\Omega\left(X^{H}, Y^{H}\right) \in$ $\mathfrak{h}$. For any $\bar{x} \in \bar{M}$, let $\mathbb{I}(\bar{x})$ the bilinear form in $\mathfrak{h}$ defined as

$$
\mathbb{I}(\bar{x})(\xi, \eta)=\bar{g}\left(\xi_{\bar{x}}^{*}, \eta_{\bar{x}}^{*}\right), \quad \forall \xi, \eta \in \mathfrak{h} .
$$

Applying Koszul's formula for $\bar{\nabla}$ and taking into account that $\left[X^{H}, \xi^{*}\right]=0$ for any $X \in \mathfrak{X}(M), \xi \in \mathfrak{h}$, we have

$$
\begin{aligned}
\bar{g}\left(\widetilde{\bar{\nabla}}_{\Omega\left(X^{H}, Y^{H}\right) *} Z^{H}, W^{H}\right) & =\bar{g}\left(\bar{\nabla}_{\Omega\left(X^{H}, Y^{H}\right)^{*}} Z^{H}, W^{H}\right)-\bar{g}\left(\bar{S}_{\Omega\left(X^{H}, Y^{H}\right)^{*}} Z^{H} W^{H}\right) \\
& =\frac{1}{2} \mathbb{I}\left(\Omega\left(X^{H}, Y^{H}\right), \Omega\left(Z^{H}, W^{H}\right)\right)-\bar{S}_{\Omega\left(X^{H}, Y^{H}\right) * Z^{H} W^{H}},
\end{aligned}
$$

where, as usual, $\widetilde{\nabla}=\bar{\nabla}-\bar{S}$. Then applying the previous equation and equation (23), a direct computation shows that

$$
\begin{aligned}
\left(\widetilde{\nabla}_{U} \widetilde{R}\right)_{X Y Z W} \circ \pi= & \left(\widetilde{\bar{\nabla}}_{U^{H}} \widetilde{\bar{R}}\right)_{X^{H} Y^{H} Z^{H} W^{H}} \\
& -\frac{1}{2} U^{H}\left(\mathbb{I}\left(\Omega\left(X^{H}, Y^{H}\right), \Omega\left(Z^{H}, W^{H}\right)\right)\right) \\
& +\frac{1}{2} \mathbb{I}\left(\Omega\left(\widetilde{\bar{\nabla}}_{U^{H}} X^{H}, Y^{H}\right), \Omega\left(Z^{H}, W^{H}\right)\right) \\
& +\frac{1}{2} \mathbb{I}\left(\Omega\left(X^{H}, \widetilde{\bar{\nabla}}_{U^{H}} Y^{H}\right), \Omega\left(Z^{H}, W^{H}\right)\right) \\
& +\frac{1}{2} \mathbb{I}\left(\Omega\left(X^{H}, Y^{H}\right), \Omega\left(\widetilde{\bar{\nabla}}_{U^{H}} Z^{H}, W^{H}\right)\right) \\
& +\frac{1}{2} \mathbb{I}\left(\Omega\left(X^{H}, Y^{H}\right), \Omega\left(Z^{H}, \widetilde{\bar{\nabla}}_{U^{H}} W^{H}\right)\right) \\
& +U^{H}\left(\bar{S}_{\Omega\left(X^{H}, Y^{H}\right)^{*} Z^{H} W^{H}}\right)-\bar{S}_{\Omega\left(\tilde{\nabla}_{U H} X^{H}, Y^{H}\right)^{*} Z^{H} W^{H}} \\
& -\bar{S}_{\Omega\left(X^{H}, \tilde{\nabla}_{U^{H}} Y^{H}\right)^{*} Z^{H} W^{H}}-\bar{S}_{\Omega\left(X^{H}, Y^{H}\right)^{*}\left(\widetilde{\nabla}_{U^{H}} Z^{H}\right) W^{H}} \\
& -\bar{S}_{\Omega\left(X^{H}, Y^{H}\right)^{*} Z^{H}\left(\tilde{\nabla}_{U^{H}} W^{H}\right)} .
\end{aligned}
$$

On the other hand, by (19)

$$
0=\left(\widetilde{\bar{\nabla}}_{X^{H}} \omega\right)\left(Y^{H}\right)-\left(\widetilde{\bar{\nabla}}_{Y^{H}} \omega\right)\left(X^{H}\right)=d \omega\left(X^{H}, Y^{H}\right)-\omega\left(\widetilde{\bar{T}}_{X^{H}} Y^{H}\right),
$$

where $\widetilde{\bar{T}}$ is the torsion tensor field of $\widetilde{\bar{\nabla}}$. Then, since by definition $\Omega(\bar{X}, \bar{Y})=$ $d \omega\left(\bar{X}^{h}, \bar{Y}^{h}\right)$, we have

$$
\Omega\left(X^{H}, Y^{H}\right)=\omega\left(\widetilde{\bar{T}}_{X^{H}} Y^{H}\right)
$$


Using that

$$
\widetilde{\bar{T}}_{X^{H}} Y^{H}=\bar{S}_{Y^{H}} X^{H}-\bar{S}_{X^{H}} Y^{H},
$$

and conditions (19) and $\widetilde{\bar{\nabla}} \bar{S}=0$, one has that

$$
\left(\widetilde{\nabla}_{U^{H}} \Omega\right)\left(X^{H}, Y^{H}\right)=\alpha\left(U^{H}\right) \cdot \Omega\left(X^{H}, Y^{H}\right) .
$$

Now, from $\omega\left(\left[X^{H}, Y^{H}\right]^{v}\right)=-\Omega\left(X^{H}, Y^{H}\right)$ and (19) we get

$$
\omega\left(\widetilde{\bar{\nabla}}_{U^{H}}\left[X^{H}, Y^{H}\right]^{v}\right)=-U^{H}\left(\Omega\left(X^{H}, Y^{H}\right)\right)+\alpha\left(U^{H}\right) \cdot \Omega\left(X^{H}, Y^{H}\right),
$$

and hence we have

$$
\begin{aligned}
U^{H}\left(\mathbb{I}\left(\Omega\left(X^{H}, Y^{H}\right), \Omega\left(Z^{H}, W^{H}\right)\right)\right) & =\bar{g}\left(\widetilde{\bar{\nabla}}_{U^{H}}\left[X^{H}, Y^{H}\right]^{v},\left[Z^{H}, W^{H}\right]^{v}\right) \\
& +\bar{g}\left(\left[X^{H}, Y^{H}\right]^{v}, \widetilde{\bar{\nabla}}_{U^{H}}\left[Z^{H}, W^{H}\right]^{v}\right) \\
& =\mathbb{I}\left(U^{H} \Omega\left(X^{H}, Y^{H}\right), \Omega\left(Z^{H}, W^{H}\right)\right) \\
& -\mathbb{I}\left(\alpha\left(U^{H}\right) \cdot \Omega\left(X^{H}, Y^{H}\right), \Omega\left(Z^{H}, W^{H}\right)\right) \\
& +\mathbb{I}\left(\Omega\left(X^{H}, Y^{H}\right), U^{H} \Omega\left(Z^{H}, W^{H}\right)\right) \\
& -\mathbb{I}\left(\Omega\left(X^{H}, Y^{H}\right), \alpha\left(U^{H}\right) \cdot \Omega\left(Z^{H}, W^{H}\right)\right) .
\end{aligned}
$$

In addition, by (25) and (26)

$$
\Omega\left(\widetilde{\bar{\nabla}}_{U^{H}} X^{H}, Y^{H}\right)+\Omega\left(X^{H}, \widetilde{\bar{\nabla}}_{U^{H}} Y^{H}\right)=-\omega\left(\widetilde{\bar{\nabla}}_{U^{H}}\left[X^{H}, Y^{H}\right]^{v}\right),
$$

so

$$
\Omega\left(\widetilde{\bar{\nabla}}_{U^{H}} X^{H}, Y^{H}\right)^{*}+\Omega\left(X^{H}, \widetilde{\bar{\nabla}}_{U^{H}} Y^{H}\right)^{*}=\widetilde{\bar{\nabla}}_{U^{H}} \Omega\left(X^{H}, Y^{H}\right)^{*}
$$

since $\widetilde{\bar{\nabla}}_{U^{H}}\left[X^{H}, Y^{H}\right]^{v}$ is vertical. Substituting the preceding formulas and grouping terms, (24) becomes

$$
\begin{aligned}
\left(\widetilde{\nabla}_{U} \widetilde{R}\right)_{X Y Z W} \circ \pi & =\left(\widetilde{\bar{\nabla}}_{U^{H}} \widetilde{\bar{R}}_{X^{H} Y^{H} Z^{H} W^{H}}\right. \\
& +\frac{1}{2} \mathbb{I}\left(\left(\widetilde{\bar{\nabla}}_{U^{H}} \Omega\right)\left(X^{H}, Y^{H}\right), \Omega\left(Z^{H}, W^{H}\right)\right) \\
& -\frac{1}{2} \mathbb{I}\left(\alpha\left(U^{H}\right) \cdot \Omega\left(X^{H}, Y^{H}\right), \Omega\left(Z^{H}, W^{H}\right)\right) \\
& +\frac{1}{2} \mathbb{I}\left(\Omega\left(X^{H}, Y^{H}\right),\left(\widetilde{\bar{\nabla}}_{U^{H}} \Omega\right)\left(Z^{H}, W^{H}\right)\right) \\
& -\frac{1}{2} \mathbb{I}\left(\Omega\left(X^{H}, Y^{H}\right), \alpha\left(U^{H}\right) \cdot \Omega\left(Z^{H}, W^{H}\right)\right) \\
& -\left(\widetilde{\bar{\nabla}}_{U^{H}} \bar{S}\right)_{\Omega\left(X^{H}, Y^{H}\right)^{*} Z^{H} W^{H}},
\end{aligned}
$$

from where, taking into account (25) and (27), we deduce that $\widetilde{\nabla}_{U} \widetilde{R}=0$. This finishes the proof of Theorem 3.7 . 
Remark 3.8 In the situation of Theorem 3.7 in the case $\bar{S}$ is a homogeneous structure tensor associated to a Lie group $\bar{G}$ acting by isometries in $\bar{M}$, one could ask if $H$ can be seen as a normal subgroup of $\bar{G}$ and if the projected tensor $S$ is associated to the group $G=\bar{G} / H$. The answer is not necessarily positive. More precisely, for a connected, simply connected and complete manifold $\bar{M}$, if we construct the group $\bar{G}$ from $\bar{S}$ following the proof of Ambrose-Singer Theorem (as in [18), one can see that the normality of $H$ is not guaranteed and the group $\bar{G}$ needs not project to the group $G$ constructed in $M$ from $S$ by the same method. An example of this situation will be shown in 4.2.1 (Hopf fibration case $\lambda=0)$.

Remark 3.9 The algebraic condition (19) for $\alpha=0$ is an invariance condition and can be implemented in Ambrose-Singer conditions as in Kiričenko's theorem (see [11). This situation can be found in the last section of the present paper in the framework of almost contact metric homogeneous structures, where this condition is automatically satisfied. Note that for non trivial $\alpha$, the situation would require an equivariant version of this theorem.

\subsection{Reduction and homogeneous classes}

In the situation of Theorem 3.7 .

Proposition 3.10 The classes $\{0\}, \mathcal{S}_{1}, \mathcal{S}_{3}, \mathcal{S}_{1} \oplus \mathcal{S}_{2}$ and $\mathcal{S}_{1} \oplus \mathcal{S}_{3}$ are invariant under the reduction procedure.

Proof. By the expression of the reduced structure tensor (20) it is obvious that if $\bar{S}=0$ then $S=0$. Let $\bar{S} \in \mathcal{S}_{1}$ given by the expression

$$
\bar{S}_{\bar{X} \bar{Y} \bar{Z}}=\bar{g}(\bar{X}, \bar{Y}) \bar{g}(\bar{\xi}, \bar{Z})-\bar{g}(\bar{Y}, \bar{\xi}) \bar{g}(\bar{X}, \bar{Z})
$$

where $\bar{\xi}$ is a vector field parallel with respect to $\widetilde{\bar{\nabla}}$. Since $\bar{S}$ is $H$-invariant the vector field $\bar{\xi}$ is also $H$-invariant, and then projectable. Let $\xi$ be the projection of $\bar{\xi}$ we have $\xi^{H}=\bar{\xi}^{h}$ and then

$$
\begin{aligned}
S_{X Y Z} \circ \pi & =\bar{g}\left(X^{H}, Y^{H}\right) \bar{g}\left(\bar{\xi}, Z^{H}\right)-\bar{g}\left(Y^{H}, \bar{\xi}\right) \bar{g}\left(X^{H}, Z^{H}\right) \\
& =\bar{g}\left(X^{H}, Y^{H}\right) \bar{g}\left(\xi^{H}, Z^{H}\right)-\bar{g}\left(Y^{H}, \xi^{H}\right) \bar{g}\left(X^{H}, Z^{H}\right) \\
& =g(X, Y) g(\xi, Z) \circ \pi-g(Y, \xi) g(X, Z) \circ \pi
\end{aligned}
$$

hence $S \in \mathcal{S}_{1}$. With a similar argument one proves that the class $\mathcal{S}_{1} \oplus \mathcal{S}_{2}$ is also invariant. For the classes $\mathcal{S}_{3}$ and $\mathcal{S}_{1} \oplus \mathcal{S}_{3}$, they are characterized by algebraic conditions clearly preserved by the reduction formula (20).

The other two classes $\mathcal{S}_{2}$ and $\mathcal{S}_{2} \oplus \mathcal{S}_{3}$ are characterized by the vanishing of the trace $c_{12}$. Let $x \in M$ and $\left\{e_{i}\right\}_{i=1, \ldots, n}$ be an orthonormal base of $T_{x} M$, then for $X \in T_{x} M$

$$
c_{12}(S)(X)=\sum_{i} S_{e_{i} e_{i} X}=\sum_{i} \bar{S}_{e_{i}^{H} e_{i}^{H} X^{H}}=c_{12}(\bar{S})\left(X^{H}\right)-\sum_{j} \bar{S}_{V_{j} V_{j} X^{H}},
$$


where $\left\{V_{j}\right\}_{j=1, \ldots, r}$ is an orthonormal basis of the vertical subspace $V_{\bar{x}} \bar{M}, \bar{x} \in$ $\pi^{-1}(x)$. From $\widetilde{\bar{\nabla}}=\widetilde{\nabla}-\bar{S}$ one has

$\bar{S}_{V_{j} V_{j} X^{H}}=\bar{g}\left(\widetilde{\nabla}_{V_{j}} V_{j}, X^{H}\right)-\bar{g}\left(\widetilde{\bar{\nabla}}_{V_{j}} V_{j}, X^{H}\right)=-\bar{g}\left(\widetilde{\nabla}_{V_{j}} X^{H}, V_{j}\right)+\bar{g}\left(\widetilde{\nabla}_{V_{j}} X^{H}, V_{j}\right)$,

where the vectors $V_{j}, j=1, \ldots, r$, are extended to unitary and respectively orthogonal vertical vector fields. As from (19) we have

$$
\omega\left(\widetilde{\bar{\nabla}}_{V_{j}} X^{H}\right)=V_{j}\left(\omega\left(X^{H}\right)\right)-\alpha\left(V_{j}\right) \cdot \omega\left(X^{H}\right)=0,
$$

the second summand in the formula for $\bar{S}_{V_{j} V_{j} X^{H}}$ is zero, and then

$$
\bar{S}_{V_{j} V_{j} X^{H}}=-\bar{g}\left(\widetilde{\nabla}_{V_{j}} X^{H}, V_{j}\right)=\bar{g}\left(B\left(V_{j}, V_{j}\right), X^{H}\right),
$$

where $B$ denotes the second fundamental form of the fibre $\pi^{-1}(x)$ at $\bar{x}$. Inserting this in (28) we obtain that

$$
c_{12}(S)(X)=c_{12}(\bar{S})\left(X^{H}\right)-\sum_{j} \bar{g}\left(B\left(V_{j}, V_{j}\right), X^{H}\right)=c_{12}(\bar{S})\left(X^{H}\right)-\bar{g}\left(\mathrm{H}, X^{H}\right)
$$

where $\mathrm{H}$ denotes the mean curvature operator (trace of $B$ ) of the fibre at $\bar{x}$. We have proved the following.

Proposition 3.11 The classes $\mathcal{S}_{2}$ and $\mathcal{S}_{2} \oplus \mathcal{S}_{3}$ are invariant under reduction if and only if the fibres of the principal bundle $\pi:(\bar{M}, \bar{g}) \rightarrow(M, g)$ are minimal Riamannian sub-manifolds of $(\bar{M}, \bar{g})$.

Remark 3.12 Proposition 3.10 and 3.11 (when the fibres are minimal) do not exclude that a homogeneous structure tensor $\bar{S}$ in a class $\mathcal{S}_{i} \oplus \mathcal{S}_{j}$ reduces to a tensor $S$ belonging to classes $\mathcal{S}_{i}$ or $\mathcal{S}_{j}$, or even to the null tensor. We shall show some examples of this situations in the next section.

\section{Examples}

\subsection{Real hyperbolic space}

The real $n$-dimensional hyperbolic space $(\mathbb{R} H(n), \bar{g})$

$$
\begin{gathered}
\mathbb{R} H(n)=\left\{\left(\bar{y}^{0}, \bar{y}^{1}, \ldots, \bar{y}^{n-1}\right) \in \mathbb{R}^{n} / \bar{y}^{0}>0\right\} \\
\bar{g}=\frac{1}{\left(\bar{y}^{0}\right)^{2}} \sum_{j=0}^{n-1} d \bar{y}^{j} \otimes d \bar{y}^{j},
\end{gathered}
$$

is a symmetric space, $\mathbb{R} H(n)=S O(n-1,1) / O(n-1)$. If we consider the Iwasawa decomposition of its full Lie group of isometries

$$
S O(1, n-1)=O(n-1) A N,
$$


then we can identify $\mathbb{R} H(n) \simeq A N$ so that the hyperbolic space has a solvable Lie group structure given by

$$
\left(\bar{x}^{0}, \bar{y}^{1}, \ldots, \bar{x}^{n-1}\right) \cdot\left(\bar{y}^{0}, \bar{y}^{1}, \ldots, \bar{y}^{n-1}\right)=\left(\bar{x}^{0} \bar{y}^{0}, \bar{x}^{0} \bar{y}^{1}+\bar{x}^{1}, \ldots, \bar{x}^{0} \bar{y}^{n-1}+\bar{x}^{n-1}\right) .
$$

Hence the real hyperbolic space acts freely, transitively and by isometries on itself by left translations. The homogeneous structure tensor $\bar{S}$ associated to this action (see [18]) is a $\mathcal{S}_{1}$ structure given by

$$
\bar{S}_{\bar{X} \bar{Y} \bar{Z}}=\bar{g}(\bar{X}, \bar{Y}) \bar{g}(\bar{\xi}, \bar{Y})-\bar{g}(\bar{\xi}, \bar{Y}) \bar{g}(\bar{X}, \bar{Z}), \quad \bar{X}, \bar{Y}, \bar{Z} \in \mathfrak{X}(\mathbb{R} H(n))
$$

where

$$
\bar{\xi}=\bar{y}^{0} \frac{\partial}{\partial \bar{y}^{0}} .
$$

Let $H_{i} \simeq \mathbb{R}, i=2, \ldots, n-1$, be the normal subgroups of $\mathbb{R} H(n)$ given by

$$
H_{i}=\{(1,0, \ldots, \lambda, 0, \ldots, 0) / \lambda \in \mathbb{R}\}
$$

where $\lambda$ is in the $i$-th position. Reduction by the action of $H_{i}$ gives the fibration

$$
\begin{aligned}
\mathbb{R} H(n) & \rightarrow \mathbb{R} H(n-1) \\
\left(\bar{y}^{0}, \ldots, \bar{y}^{n-1}\right) & \mapsto\left(\bar{y}^{0}, \ldots, \bar{y}^{i-1}, \bar{y}^{i+1} \ldots, \bar{y}^{n-1}\right)
\end{aligned}
$$

with vertical and horizontal subspaces at $\bar{y} \in \mathbb{R} H(n)$

$$
\begin{aligned}
& V_{\bar{y}} \mathbb{R} H(n)=\operatorname{span}\left\{\frac{\partial}{\partial \bar{y}^{i}}\right\}, \\
& H_{\bar{y}} \mathbb{R} H(n)=\operatorname{span}\left\{\frac{\partial}{\partial \bar{y}^{0}}, \ldots, \frac{\partial}{\partial \bar{y}^{i-1}}, \frac{\partial}{\partial \bar{y}^{i+1}}, \ldots, \frac{\partial}{\partial \bar{y}^{n-1}}\right\} .
\end{aligned}
$$

Hence the induced metric on $\mathbb{R} H(n-1)$ is

$$
g=\frac{1}{\left(y^{0}\right)^{2}} \sum_{j=0}^{n-2} d y^{j} \otimes d y^{j}
$$

where $\left(y^{0}, \ldots, y^{n-2}\right)$ are the natural coordinates of $\mathbb{R} H(n-1)$. As a straightforward computation shows, the reduced homogeneous structure tensor $S$ is

$$
S_{X Y Z}=g(X, Y) g(\xi, Z)-g(\xi, Y) g(X, Z), \quad X, Y, Z \in \mathfrak{X}(\mathbb{R} H(n-1))
$$

where

$$
\xi=y^{0} \frac{\partial}{\partial y^{0}} .
$$

We have proved that the reduction $\mathbb{R} H(n) \rightarrow \mathbb{R} H(n-1)$ sends the canonical tensor associated to the solvable structure of the $n$-dimensional hyperbolic space to the canonical tensor associated to the solvable structure of the $n-1$ dimensional hyperbolic space. The reduction procedure has then preserved the $\mathcal{S}_{1}$ class in this case. 
We now confine ourselves to the 4-dimensional hyperbolic space. Besides its symmetric description, all other groups of isometries acting transitively are of the type (cf. [5]) $\bar{G}=F N$, where $F$ is a connected closed subgroup of $S O(3) A$ with nontrivial projection to $A$. In particular, we now consider

$$
\bar{G}=S O(2) A N
$$

Geometrically, if we see $S O(2)$ as the isotropy group of the point $\bar{x}=(1,0,0,0)$, its Lie algebra $\overline{\mathfrak{k}}$ are infinitesimal rotations generated by

$$
r=\bar{y}^{2} \frac{\partial}{\partial \bar{y}^{3}}-\bar{y}^{3} \frac{\partial}{\partial \bar{y}^{2}} \text {. }
$$

The subspace $\overline{\mathfrak{m}}=\mathfrak{a} \oplus \mathfrak{n}$, which is the lie algebra of the factor $A N$, gives a reductive decomposition

$$
\overline{\mathfrak{g}}=\overline{\mathfrak{m}} \oplus \overline{\mathfrak{k}}
$$

Let $a \in \mathfrak{a}, n_{1}, n_{2}, n_{3} \in \mathfrak{n}$ be the generators of $\mathfrak{a}$ and $\mathfrak{n}$ respectively, where $n_{i}$ is the infinitesimal translation in $\mathbb{R} H(4)$ in the direction of $\partial / \partial \bar{y}^{i}$. All other reductive decompositions $\overline{\mathfrak{g}}=\overline{\mathfrak{m}}^{\varphi}+\overline{\mathfrak{k}}$ associated to $\overline{\mathfrak{g}}$ and $\overline{\mathfrak{k}}$ are given by the graph of any equivariant map $\varphi: \mathfrak{m} \rightarrow \mathfrak{k}$. As a computation shows, all these equivariant maps are

$$
\begin{aligned}
\varphi_{\left(\lambda_{0}, \lambda_{1}\right)}: & \mathfrak{m} \\
a & \mapsto \mathfrak{k} \\
n_{1} & \mapsto \lambda_{0} r \\
n_{2}, n_{3} & \mapsto \lambda_{1} r
\end{aligned}
$$

with $\lambda_{0}, \lambda_{1} \in \mathbb{R}$. The homogeneous structure tensors associated to this 2parameter family of reductive decompositions are

$$
\bar{S}^{\left(\lambda_{0}, \lambda_{1}\right)}=\frac{1}{\left(\bar{y}^{0}\right)^{3}}\left(\sum_{k=1}^{3} d \bar{y}^{k} \otimes d \bar{y}^{k} \wedge d \bar{y}^{0}-\lambda_{0} d \bar{y}^{0} \otimes d \bar{y}^{2} \wedge d \bar{y}^{3}-\lambda_{1} d \bar{y}^{1} \otimes d \bar{y}^{2} \wedge d \bar{y}^{3}\right)
$$

and the canonical connection $\widetilde{\widetilde{\nabla}}=\bar{\nabla}-\bar{S}^{\left(\lambda_{0}, \lambda_{1}\right)}$ (where $\bar{\nabla}$ is the Levi-Civita connection of $\bar{g}$ ) is then given by

$$
\begin{gathered}
\widetilde{\bar{\nabla}}_{\partial_{0}} \partial_{0}=-\frac{1}{\bar{y}^{0}} \partial_{0}, \quad \widetilde{\bar{\nabla}}_{\partial_{0}} \partial_{1}=-\frac{1}{\bar{y}^{0}} \partial_{1}, \quad \widetilde{\bar{\nabla}}_{\partial_{0}} \partial_{2}=-\frac{1}{\bar{y}^{0}} \partial_{2}+\frac{\lambda_{0}}{\bar{y}^{0}} \partial_{3}, \\
\widetilde{\widetilde{\nabla}}_{\partial_{0}} \partial_{3}=-\frac{1}{\bar{y}^{0}} \partial_{3}-\frac{\lambda_{0}}{\bar{y}^{0}} \partial_{2}, \quad \widetilde{\bar{\nabla}}_{\partial_{1}} \partial_{2}=\frac{\lambda_{1}}{\bar{y}^{0}} \partial_{3}, \quad \widetilde{\bar{\nabla}}_{\partial_{1}} \partial_{3}=-\frac{\lambda_{1}}{\bar{y}^{0}} \partial_{2},
\end{gathered}
$$

where $\partial_{k}$ stands for $\frac{\partial}{\partial \bar{y}^{k}}$. Let $H \simeq \mathbb{R}$ be the subgroup of $\mathbb{R} H(4)$ given by

$$
H=\{(1, \lambda, 0,0) / \lambda \in \mathbb{R}\} .
$$

We take the $H$-principal bundle

$$
\begin{aligned}
\mathbb{R} H(4) & \rightarrow \mathbb{R} H(3) \\
\left(\bar{y}^{0}, \bar{y}^{1}, \bar{y}^{2}, \bar{y}^{3}\right) & \mapsto\left(\bar{y}^{0}, \bar{y}^{2}, \bar{y}^{3}\right)
\end{aligned}
$$


with mechanical connection form $\omega=d \bar{y}^{1}$. We have that

$$
\widetilde{\bar{\nabla}} \omega=\left(\frac{1}{\bar{y}^{0}} d \bar{y}^{0}\right) \cdot \omega
$$

where we have identified $\mathfrak{h} \simeq \mathbb{R}$ and $\operatorname{End}(\mathfrak{h}) \simeq \mathbb{R}$. From Theorem 3.7, the family of homogeneous structure tensors $\bar{S}^{\left(\lambda_{0}, \lambda_{1}\right)}$ can then be reduced to $\mathbb{R} H(3)$. If $\left(y^{0}, y^{1}, y^{2}\right)$ are the standard coordinates of $\mathbb{R} H(3)$, these reduced homogeneous structure tensors form a one-parameter family

$$
S^{\lambda_{0}}=\frac{1}{\left(y^{0}\right)^{3}}\left(\sum_{k=1}^{2} d y^{k} \otimes d y^{k} \wedge d y^{0}-\lambda_{0} d y^{0} \otimes d y^{1} \wedge d y^{2}\right) .
$$

Note that in the expression of both $\bar{S}^{\left(\lambda_{0}, \lambda_{1}\right)}$ and $S^{\lambda_{0}}$ the first summand is the standard $\mathcal{S}_{1}$ structure of $\mathbb{R} H(4)$ and $\mathbb{R} H(3)$ respectively. The other summands are of type $\mathcal{S}_{2} \oplus \mathcal{S}_{3}$ since they have null trace, which makes $\bar{S}^{\left(\lambda_{0}, \lambda_{1}\right)}$ and $S^{\lambda_{0}}$ of type $\mathcal{S}_{1} \oplus \mathcal{S}_{2} \oplus \mathcal{S}_{3}$ in the generic case. In the especial case $\lambda_{0}=0$ we will have a reduction of the generic class $\mathcal{S}_{1} \oplus \mathcal{S}_{2} \oplus \mathcal{S}_{3}$ to the class $\mathcal{S}_{1}$. This example can be generalized to the principal bundle $\mathbb{R} H(n) \rightarrow \mathbb{R} H(n-1)$.

\subsection{Hopf Fibrations}

\subsubsection{The fibration $S^{3} \rightarrow S^{2}$}

Let $S^{3} \subset \mathbb{R}^{4} \simeq \mathbb{C}^{2}$ be the 3-sphere with its standard Riemannian metric with full isometry group $O(4)$. The natural action of $U(2)$ in $\mathbb{C}^{2}$ defines a transitive and effective action of $U(2)$ on $S^{3}$ given by

$$
\begin{aligned}
& U(2) \quad \hookrightarrow \quad S O(4) \\
& \left(\begin{array}{ll}
a & b \\
c & d
\end{array}\right) \mapsto\left(\begin{array}{cccc}
\operatorname{Re}(a) & -\operatorname{Im}(a) & \operatorname{Re}(b) & -\operatorname{Im}(b) \\
\operatorname{Im}(a) & \operatorname{Re}(a) & \operatorname{Im}(b) & \operatorname{Re}(b) \\
\operatorname{Re}(c) & -\operatorname{Im}(c) & \operatorname{Re}(d) & -\operatorname{Im}(d) \\
\operatorname{Im}(c) & \operatorname{Re}(c) & \operatorname{Im}(d) & \operatorname{Re}(d)
\end{array}\right)
\end{aligned}
$$

The isotropy group at $\bar{x}=(1,0,0,0) \in S^{3}$ is

$$
\bar{K}=\left\{\left(\begin{array}{ll}
1 & 0 \\
0 & z
\end{array}\right) \in U(2) / z \in U(1)\right\}
$$

with lie algebra

$$
\overline{\mathfrak{k}}=\operatorname{span}\left\{\left(\begin{array}{ll}
0 & 0 \\
0 & i
\end{array}\right)\right\} .
$$

It is easy to see that the complement

$$
\overline{\mathfrak{m}}=\operatorname{span}\left\{\left(\begin{array}{cc}
0 & 1 \\
-1 & 0
\end{array}\right),\left(\begin{array}{cc}
0 & i \\
i & 0
\end{array}\right),\left(\begin{array}{cc}
i & 0 \\
0 & -i
\end{array}\right)\right\}
$$

makes $\mathfrak{u}(2)=\overline{\mathfrak{m}} \oplus \overline{\mathfrak{k}}$ a reductive decomposition. The rest of complements $\overline{\mathfrak{m}}^{\prime}$ giving reductive decompositions $\mathfrak{u}(2)=\overline{\mathfrak{m}}^{\prime} \oplus \overline{\mathfrak{k}}$ are obtained as the graph of 
$\operatorname{Ad}(\bar{K})$-equivariant maps $\varphi: \overline{\mathfrak{m}} \rightarrow \overline{\mathfrak{k}}$. One can check that these decompositions are exhausted by the following one-parameter family of complements

$$
\overline{\mathfrak{m}}_{\lambda}=\operatorname{span}\left\{\left(\begin{array}{cc}
0 & 1 \\
-1 & 0
\end{array}\right),\left(\begin{array}{cc}
0 & i \\
i & 0
\end{array}\right),\left(\begin{array}{cc}
i & 0 \\
0 & -i
\end{array}\right)+\lambda\left(\begin{array}{cc}
0 & 0 \\
0 & i
\end{array}\right)\right\}, \quad \lambda \in \mathbb{R} .
$$

From formula (14), the expression of the homogeneous structure tensor $\bar{S}^{\lambda}$ associated to each reductive decomposition computed at $T_{\bar{x}} S^{3}$ is given by

$$
\left(\bar{S}^{\lambda}\right)_{\bar{x}}=(\lambda-1) d \bar{x}^{2} \otimes d \bar{x}^{3} \wedge d \bar{x}^{4}+d \bar{x}^{3} \otimes d \bar{x}^{2} \wedge d \bar{x}^{4}-d \bar{x}^{4} \otimes d \bar{x}^{2} \wedge d \bar{x}^{3},
$$

where $\left(\bar{x}^{1}, \bar{x}^{2}, \bar{x}^{3}, \bar{x}^{4}\right)$ is the natural system of coordinates in $\mathbb{R}^{4}$.

Let $H$ be the subgroup of $U(2)$ isomorphic to $U(1)$ given by

$$
H=\left\{\left(\begin{array}{cc}
z & 0 \\
0 & z
\end{array}\right) / z \in U(1)\right\}
$$

It is easy to check that $H$ is a normal subgroup of $U(2)$ acting freely on $S^{3}$. Reduction by the action of $H$ gives the Hopf fibration $S^{3} \rightarrow S^{2}$ with vertical and horizontal subspaces at $\bar{x}$

$$
V_{\bar{x}} S^{3}=\operatorname{span}\left\{\frac{\partial}{\partial \bar{x}_{2}}\right\}, \quad H_{\bar{x}} S^{3}=\operatorname{span}\left\{\frac{\partial}{\partial \bar{x}_{3}}, \frac{\partial}{\partial \bar{x}_{4}}\right\} .
$$

Since all the terms of $\bar{S}^{\lambda}$ have the vertical factor $d \bar{x}^{2}$, it is obvious that they all reduce to the structure tensor $S=0$ on $S^{2}$, describing $S^{2}$ as a symmetric space. Note that this is what one can expect since $S^{2}$ only admits the zero homogeneous structure tensor [18].

For the case $\lambda=0$ one can follow the proof of Ambrose-Singer's Theorem to construct the Lie algebra of a group acting transitively on $S^{3}$. As a computation shows the holonomy of the connection $\widetilde{\bar{\nabla}}=\bar{\nabla}-\bar{S}_{0}$ is trivial, and one obtains the reductive decomposition $T_{e} S^{3} \oplus\{0\} \simeq \mathfrak{s u}(2)$ which describes the action of $S U(2) \simeq S^{3}$ on itself. We then have an example of a homogeneous Riemannian structure $\bar{S}^{0}$ satisfying $\widetilde{\bar{\nabla}} \omega=\alpha \cdot \omega$ as in Theorem 3.7 ( $\omega$ being the mechanical connection form of the Hopf fibration $S^{3} \rightarrow S^{2}$ ), but for which the structure group of the fibration $(H=U(1))$ can not be seen as a normal subgroup of the group $\left(\bar{G}^{\prime}=S U(2)\right)$ obtained by the proof of Ambrose-Singer's Theorem .

Remark 4.1 There are not more reducible tensors than those described above as the other groups acting transitively on $S^{3}$ are $S O(4)$, which has no normal subgroups, and $S U(2) \simeq S^{3}$. In addition, this procedure can be adapted to the Berger 3-spheres, where a family of homogeneous structures is calculated in (9]). All reducible structures of this family reduce to $S=0$ on $S^{2}$ as expected.

Remark 4.2 The groups acting isometrically and transitively on $S^{7}$ (see [17) are $S O(7), S U(4), S p(2) S p(1), U(4)$ and $S p(2) U(1)$. The first two groups do not 
have normal subgroups and hence do not fit in the reduction scheme. The group $\bar{G}=S p(2) S p(1)$ has the normal subgroup $H=S p(1)=S U(2)$, which gives the Hopf fibration $S^{7} \rightarrow S^{4}$. In this case, a similar computation to the fibration $S^{3} \rightarrow S^{2}$ shows that the corresponding homogeneous Riemannian structures in the 7-sphere reduce to the null tensor on $S^{4}$, the only homogeneous structure in the four dimensional sphere. The last two groups are analized in the following subsection.

\subsubsection{The fibration $S^{7} \rightarrow \mathbb{C} P^{3}$}

Let $\Delta_{j}^{i}$ denote the $4 \times 4$ complex matrix with 1 in the $i$-th row and the $j$-th column and the rest zeros. Let $S^{7}$ be the standard 7 -sphere as a Riemannian sub-manifold of $\mathbb{C}^{4}$ with the usual Hermitian inner product. The standard action of the unitary group $U(4)$ on $\mathbb{C}^{4}$ gives a transitive and effective action on $S^{7}$ by isometries. The isotropy group $\bar{K}$ at $\bar{x}=(1,0,0,0) \in \mathbb{S}^{7}$ is isomorphic to $U(3)$ and we can decompose $\mathfrak{u}(4)=\overline{\mathfrak{m}} \oplus \overline{\mathfrak{k}}$ where

$$
\overline{\mathfrak{k}}=\left\{\left(\begin{array}{ll}
0 & 0 \\
0 & A
\end{array}\right) / A \in \mathfrak{u}(3)\right\}
$$

and

$$
\overline{\mathfrak{m}}=\operatorname{span}\left\{i \Delta_{1}^{1}, \Delta_{j}^{1}-\Delta_{1}^{j}, i\left(\Delta_{j}^{1}+\Delta_{1}^{j}\right), j=1,2,3\right\} .
$$

One can check that $\mathfrak{u}(4)=\overline{\mathfrak{m}} \oplus \overline{\mathfrak{k}}$ is the unique reductive decomposition of $\mathfrak{u}(4)$ with respect to $\overline{\mathfrak{k}}$. From (14), identifying $\mathbb{R}^{8} \simeq \mathbb{C}^{4}$ and taking its natural coordinates $\left(\bar{x}^{1}, \ldots, \bar{x}^{8}\right)$, the expression of the homogeneous structure tensor $\bar{S}$ associated to this decomposition at $T_{\bar{x}} S^{7}$ reads

$$
\begin{aligned}
\bar{S}_{\bar{x}}= & d \bar{x}^{3} \otimes d \bar{x}^{2} \wedge d \bar{x}^{4}-d \bar{x}^{4} \otimes d \bar{x}^{2} \wedge d \bar{x}^{3}+d \bar{x}^{5} \otimes d \bar{x}^{2} \wedge d \bar{x}^{6} \\
& -d \bar{x}^{6} \otimes d \bar{x}^{2} \wedge d \bar{x}^{5}+d \bar{x}^{7} \otimes d \bar{x}^{2} \wedge d \bar{x}^{8}-d \bar{x}^{8} \otimes d \bar{x}^{2} \wedge d \bar{x}^{7}
\end{aligned}
$$

As a simple computation shows, this tensor belongs to the class $\mathcal{S}_{2} \oplus \mathcal{S}_{3}$.

Let $H$ be the subgroup of $U(4)$ isomorphic to $U(1)$ given by

$$
H=\{z \cdot I / z \in U(1)\}
$$

where $I$ is the $4 \times 4$ identity matrix. It is obvious that $H$ is a normal subgroup of $U(4)$ the action of which on $S^{7}$ is free. The reduction of $S^{7}$ by the action of $H$ gives the Hopf fibration $S^{7} \rightarrow \mathbb{C} P^{3}$ with which the complex projective space inherits the Fubiny-Study metric. The vertical and horizontal subspaces at $\bar{x}$ are

$$
V_{\bar{x}} S^{7}=\operatorname{span}\left\{\frac{\partial}{\partial \bar{x}_{2}}\right\}, \quad H_{\bar{x}} S^{7}=\operatorname{span}\left\{\frac{\partial}{\partial \bar{x}_{3}}, \ldots, \frac{\partial}{\partial \bar{x}_{8}}\right\} .
$$

As in the Hopf fibration $S^{3} \rightarrow S^{2}$, the homogeneous structure tensor $\bar{S}$ reduces to $S=0$, describing

$$
\mathbb{C} P^{3}=\frac{U(4)}{U(3) \times U(1)}
$$


as a symmetric space.

If $\mathbb{H}$ denotes the quaternion algebra, we now see the 7 -sphere

$$
S^{7}=\left\{\left(\begin{array}{l}
q_{1} \\
q_{2}
\end{array}\right) \in \mathbb{H}^{2} /\left|q_{1}\right|^{2}+\left|q_{2}\right|^{2}=1\right\}
$$

as a Riemannian sub-manifold of $\mathbb{H}^{2}$ with the standard quaternion inner product. The group $S p(2) U(1)=S p(2) \times_{\mathbb{Z}_{2}} U(1)$ acts on $\mathbb{H}^{2}$ by

$$
(A, z) \cdot\left(\begin{array}{l}
q_{1} \\
q_{2}
\end{array}\right)=A\left(\begin{array}{l}
q_{1} \bar{z} \\
q_{2} \bar{z}
\end{array}\right), \quad\left(\begin{array}{l}
q_{1} \\
q_{2}
\end{array}\right) \in \mathbb{H}^{2}, A \in S p(2), z \in U(1)
$$

where $\bar{z}$ stands for the complex conjugation. This action restricts to a transitive and effective action by isometries on $S^{7}$. The isotropy group at $\bar{x}=(1,0) \in S^{7}$ is

$$
\bar{K}=\left\{\left(\left(\begin{array}{cc}
z & 0 \\
0 & q
\end{array}\right), z\right) / q \in S p(1), z \in U(1)\right\} / \mathbb{Z}_{2}
$$

which is isomorphic to $S p(1) U(1)$. Let $i, j, k$ be the imaginary quaternion units and $i$ be the imaginary complex unit. Then, the Lie algebra of $S p(2) U(1)$ is $\mathfrak{s p}(2) \oplus \mathfrak{u}(1)$ where

$$
\begin{aligned}
\mathfrak{s p}(2)=\operatorname{span}\{ & \left(\begin{array}{cc}
0 & 1 \\
-1 & 0
\end{array}\right),\left(\begin{array}{ll}
i & 0 \\
0 & 0
\end{array}\right),\left(\begin{array}{ll}
0 & i \\
i & 0
\end{array}\right),\left(\begin{array}{ll}
j & 0 \\
0 & 0
\end{array}\right),\left(\begin{array}{ll}
0 & j \\
j & 0
\end{array}\right) \\
& \left.\left(\begin{array}{ll}
k & 0 \\
0 & 0
\end{array}\right),\left(\begin{array}{ll}
0 & k \\
k & 0
\end{array}\right),\left(\begin{array}{ll}
0 & 0 \\
0 & i
\end{array}\right),\left(\begin{array}{ll}
0 & 0 \\
0 & j
\end{array}\right),\left(\begin{array}{ll}
0 & 0 \\
0 & k
\end{array}\right)\right\}
\end{aligned}
$$

and $\mathfrak{u}(1)=\operatorname{span}\{i\}$; and then the isotropy algebra is

$$
\overline{\mathfrak{k}}=\operatorname{span}\left\{\left(\begin{array}{ll}
i & 0 \\
0 & 0
\end{array}\right)+i,\left(\begin{array}{ll}
0 & 0 \\
0 & i
\end{array}\right),\left(\begin{array}{ll}
0 & 0 \\
0 & j
\end{array}\right),\left(\begin{array}{ll}
0 & 0 \\
0 & k
\end{array}\right)\right\} .
$$

Taking

$$
\begin{aligned}
\overline{\mathfrak{m}}= & \operatorname{span}\left\{\left(\begin{array}{cc}
0 & 1 \\
-1 & 0
\end{array}\right),\left(\begin{array}{ll}
i & 0 \\
0 & 0
\end{array}\right),\left(\begin{array}{ll}
0 & i \\
i & 0
\end{array}\right)\right. \\
& \left.\left(\begin{array}{ll}
j & 0 \\
0 & 0
\end{array}\right),\left(\begin{array}{ll}
0 & j \\
j & 0
\end{array}\right),\left(\begin{array}{ll}
k & 0 \\
0 & 0
\end{array}\right),\left(\begin{array}{ll}
0 & k \\
k & 0
\end{array}\right)\right\}
\end{aligned}
$$

we have that $\mathfrak{s p}(2) \oplus \mathfrak{u}(1)=\overline{\mathfrak{m}} \oplus \overline{\mathfrak{k}}$ is a reductive decomposition. All other reductive decompositions associated to $\mathfrak{s p}(2) \oplus \mathfrak{u}(1)$ and $\overline{\mathfrak{k}}$ are given by a oneparameter family of complements $\overline{\mathfrak{m}}_{\lambda}, \lambda \in \mathbb{R}$, which are the graph of the $A d(\bar{K})$ equivariant maps $\varphi_{\lambda}: \overline{\mathfrak{m}} \rightarrow \overline{\mathfrak{k}}$, where $\varphi_{\lambda} \operatorname{maps}\left(\begin{array}{ll}i & 0 \\ 0 & 0\end{array}\right)$ to $\lambda\left(\begin{array}{ll}i & 0 \\ 0 & 0\end{array}\right)+\lambda i$ and the rest of elements of the basis to zero. Identifying $\mathbb{H}^{2} \equiv \mathbb{R}^{8}$, the homogeneous 
structure tensor $\bar{S}^{\lambda}$ associated to each reductive decomposition $\mathfrak{s p}(2) \oplus \mathfrak{u}(1)=$ $\overline{\mathfrak{m}}_{\lambda} \oplus \overline{\mathfrak{k}}$ is computed at $T_{\bar{x}} S^{7}$ as

$$
\begin{aligned}
\left(\bar{S}^{\lambda}\right)_{\bar{x}}= & d \bar{x}^{5} \otimes d \bar{x}^{2} \wedge d \bar{x}^{6}+d \bar{x}^{5} \otimes d \bar{x}^{3} \wedge d \bar{x}^{7}+d \bar{x}^{5} \otimes d \bar{x}^{4} \wedge d \bar{x}^{8} \\
& -\lambda d \bar{x}^{2} \otimes d \bar{x}^{5} \wedge d \bar{x}^{6}+(1+2 \lambda) d \bar{x}^{2} \otimes d \bar{x}^{3} \wedge d \bar{x}^{4}+\lambda d \bar{x}^{2} \otimes d \bar{x}^{7} \wedge d \bar{x}^{8} \\
& +d \bar{x}^{6} \otimes d \bar{x}^{5} \wedge d \bar{x}^{2}+d \bar{x}^{6} \otimes d \bar{x}^{3} \wedge d \bar{x}^{8}-d \bar{x}^{6} \otimes d \bar{x}^{4} \wedge d \bar{x}^{7} \\
& +d \bar{x}^{3} \otimes d \bar{x}^{2} \wedge d \bar{x}^{4}+d \bar{x}^{4} \otimes d \bar{x}^{2} \wedge d \bar{x}^{3} \\
& -d \bar{x}^{7} \otimes d \bar{x}^{3} \wedge d \bar{x}^{5}-d \bar{x}^{7} \otimes d \bar{x}^{2} \wedge d \bar{x}^{8}+d \bar{x}^{7} \otimes d \bar{x}^{4} \wedge d \bar{x}^{6} \\
& -d \bar{x}^{8} \otimes d \bar{x}^{4} \wedge d \bar{x}^{5}+d \bar{x}^{8} \otimes d \bar{x}^{2} \wedge d \bar{x}^{7}-d \bar{x}^{8} \otimes d \bar{x}^{3} \wedge d \bar{x}^{6} .
\end{aligned}
$$

Let $H=\{(I d, w) / w \in U(1)\} \subset S p(2) U(1)$, where $I d$ is the identity of $S p(2)$, it is easy to see that $H$ is a normal subgroup of $S p(2) U(1)$ isomorphic to $U(1)$.

Reduction by the action of $H$ gives again the Hopf fibration $\pi: S^{7} \rightarrow \mathbb{C} P^{3}$ with $\pi(\bar{x})=[1: 0: 0: 0] \in \mathbb{C} P^{3}$. The vertical and horizontal subspaces of $\pi$ at $\bar{x}$ are

$$
V_{\bar{x}} S^{7}=\operatorname{span}\left\{\frac{\partial}{\partial \bar{x}_{2}}\right\}, \quad H_{\bar{x}} S^{7}=\operatorname{span}\left\{\frac{\partial}{\partial \bar{x}_{3}}, \ldots, \frac{\partial}{\partial \bar{x}_{8}}\right\}
$$

Let $\left(t^{1}, \ldots, t^{6}\right): \mathbb{C} P^{3}-\left\{z_{0}=0\right\} \rightarrow \mathbb{R}^{6}$ be the coordinate system around $x=[1: 0: 0: 0]$ given by

$$
\left[z_{0}: z_{1}: z_{2}: z_{3}\right] \mapsto\left(\operatorname{Re}\left(\frac{z_{1}}{z_{0}}\right), \operatorname{Im}\left(\frac{z_{1}}{z_{0}}\right), \operatorname{Re}\left(\frac{z_{2}}{z_{0}}\right), \operatorname{Im}\left(\frac{z_{2}}{z_{0}}\right), \operatorname{Re}\left(\frac{z_{3}}{z_{0}}\right), \operatorname{Im}\left(\frac{z_{3}}{z_{0}}\right)\right) .
$$

The reduced homogeneous structure tensor $S$ is computed at $T_{x} \mathbb{C} P^{3}$ as

$$
\begin{aligned}
S_{x} & =d t^{3} \otimes d t^{1} \wedge d t^{5}+d t^{3} \otimes d t^{2} \wedge d t^{6} \\
& +d t^{4} \otimes d t^{1} \wedge d t^{6}-d t^{4} \otimes d t^{2} \wedge d t^{5} \\
& +d t^{5} \otimes d t^{2} \wedge d t^{4}-d t^{5} \otimes d t^{1} \wedge d t^{3} \\
& -d t^{6} \otimes d t^{2} \wedge d t^{3}-d t^{6} \otimes d t^{1} \wedge d t^{4}
\end{aligned}
$$

It is easy to check that $\bar{S}^{\lambda}$ is a $\mathcal{S}_{2} \oplus \mathcal{S}_{3}$ structure for all $\lambda \in \mathbb{R}$ which is not $\mathcal{S}_{2}$ nor $\mathcal{S}_{3}$ for any $\lambda$, and $S$ is also a strict $\mathcal{S}_{2} \oplus \mathcal{S}_{3}$ structure. Note that in the latter and the previous example the class $\mathcal{S}_{2} \oplus \mathcal{S}_{3}$ is preserved by the reduction procedure. This fact is expected from Proposition 3.11 since the fibres of the Hopf fibration are totally geodesic and in particular minimal Riemannian sub-manifolds of $S^{7}$.

\section{Almost contact metric-almost Hermitian and Sasakiann-Kähler reduction}

An almost contact structure on a manifold $\bar{M}$ is a triple $(\phi, \xi, \eta)$ where $\phi$ is a $(1,1)$-tensor field, $\xi$ is a vector field, and $\eta$ is a 1 -form satisfying

$$
\begin{gathered}
\phi(\xi)=0, \quad \eta(\phi(\bar{X}))=0, \quad \eta(\xi)=1, \\
\phi^{2}=-\mathrm{id}+\eta \otimes \xi,
\end{gathered}
$$


for all $\bar{X} \in \mathfrak{X}(\bar{M})$. The almost contact structure is said to be strictly regular if $\xi$ is a regular vector field such that all orbits of which are homeomorphic, and invariant if $\phi$ and $\eta$ are invariant by the action of the one parameter group of $\xi$. In the following all almost contact structures are supposed to be invariant and strictly regular. In [16] the following results are proved:

Theorem 5.1 Let $(\phi, \xi, \eta)$ be an almost contact structure and $M$ the space of orbits given by $\xi$. Then $M$ is endowed with a smooth structure such that $\pi: \bar{M} \rightarrow M$ is an principal bundle and $\eta$ is a connection form.

Theorem 5.2 In the situation of the previous Theorem, the $(1,1)$-tensor field $J$ defined in $M$ by

$$
J_{x} X=\pi_{*}\left(\phi_{\bar{x}} X^{H}\right), \quad x \in M, X \in \mathfrak{X}(M),
$$

where $\bar{x} \in \pi^{-1}(x) \subset \bar{M}$ and $X^{H}$ is the horizontal lift of $X$ with respect to $\eta$, is an almost complex structure.

If $\bar{M}$ is equipped with a Riemannian metric $\bar{g}$, an almost contact structure $(\phi, \xi, \eta)$ is said to be metric if the following conditions hold

$$
\bar{g}(\xi, \bar{X})=\eta(\bar{X}), \quad \bar{g}(\phi \bar{X}, \phi \bar{Y})=\bar{g}(\bar{X}, \bar{Y})+\eta(\bar{X}) \eta(\bar{Y}) .
$$

Note that this implies that $\eta$ defines the mechanical connection in $(\bar{M}, \bar{g}) \rightarrow M$ and induces a Riemannian metric $g$ in $M$. In this situation it can be proved [16] that $(J, g)$ is almost Hermitian. Let $\Phi(\bar{X}, \bar{Y})=\bar{g}(\phi \bar{X}, \bar{Y})$ be the fundamental 2 -form of the almost contact metric structure, then $(\phi, \xi, \eta, g)$ is called an almost Sasakian structure if $d \eta=2 \Phi$. If moreover $\bar{\nabla} \phi=\bar{g} \otimes \xi-$ id $\otimes \eta$ where $\bar{\nabla}$ is the Levi-Civita connection of $\bar{g}$, then it is called a Sasakian structure. It can be proved [16] that if $(\phi, \xi, \eta, g)$ is (almost) Sasakian then $(J, g)$ is (almost) Kähler.

An almost contact metric manifold is called homogeneous almost contact metric if there is a transitive group of isometries such that $\phi$ is invariant (and then also $\xi$ and $\eta$ ). If the manifold is (almost) Sasakian then it is called (almost) Sasakian homogeneous. A homogeneous structure tensor $\bar{S}$ on $\bar{M}$ is called a homogeneous almost contact metric structure if $\widetilde{\bar{\nabla}} \phi=0$ (and then $\widetilde{\bar{\nabla}} \xi=0$ and $\widetilde{\widetilde{\nabla}} \eta=0$ ). From the result of Kiričenko [11] we have that a connected, simply connected and complete Riemannian manifold is a homogeneous almost contact metric manifold if and only if it admits a homogeneous almost contact metric structure. If the manifold is (almost) Sasakian then it is homogeneous (almost) Sasakian if and only if it admits a homogeneous (almost) Sasakian structure.

We now assume that $\bar{S}$ is an almost contact metric homogeneous structure invariant by the one parameter group of $\xi$. Since $\widetilde{\widetilde{\nabla}} \eta=0$, we are in the situation of Theorem 3.7 and then the tensor $S_{X} Y=\pi_{*}\left(\bar{S}_{X^{H}} Y^{H}\right)$ defines a homogenous structure on $M$.

Proposition 5.3 The reduced homogeneous structure $S$ in $M$ is a homogeneous almost Hermitian structure on $M$. Moreover, if $\bar{S}$ is homogeneous (almost) Sasakian structure, then the reduced homogeneous structure $S$ is a homogeneous (almost) Kähler structure on $M$. 
Proof. Let $\widetilde{\nabla}=\nabla-S$, where $\nabla$ is the Levi-Civita connection of $g$. Then $\widetilde{\nabla}_{X} Y=\pi_{*}\left(\widetilde{\bar{\nabla}}_{X^{H}} Y^{H}\right)$. Since $\eta(\phi(\bar{X}))=0$ we have that $\phi(\bar{X})$ is horizontal for all $\bar{X} \in \mathfrak{X}(\bar{M})$. For any $X, Y \in \mathfrak{X}(M)$ we have

$$
\begin{aligned}
\left(\widetilde{\nabla}_{X} J\right) Y & =\widetilde{\nabla}_{X}(J Y)-J\left(\widetilde{\nabla}_{X} Y\right) \\
& =\pi_{*}\left(\widetilde{\bar{\nabla}}_{X^{H}}(J Y)^{H}\right)-\pi_{*}\left(\phi\left(\widetilde{\bar{\nabla}}_{X^{H}} Y^{H}\right)\right) \\
& =\pi_{*}\left(\widetilde{\bar{\nabla}}_{X^{H}}\left(\phi Y^{H}\right)-\phi\left(\widetilde{\bar{\nabla}}_{X^{H}} Y^{H}\right)\right) \\
& =\pi_{*}\left(\left(\widetilde{\bar{\nabla}}_{X^{H}} \phi\right) Y^{H}\right) \\
& =0
\end{aligned}
$$

and hence $\widetilde{\nabla} J=0$.

We now apply Proposition 5.3 to the Hopf fibrations $S^{3} \rightarrow S^{2}$ and $S^{7} \rightarrow$ $\mathbb{C} P^{3}$ and check that the Sasakian-Kähler reduction procedure gives the null Kähler structures of the reduced spaces, the only homogeneous Kähler structures existing on $S^{2}$ and $\mathbb{C} P^{3}$. For the first case, let $\left(\bar{x}^{1}, \bar{x}^{2}, \bar{x}^{3}, \bar{x}^{4}\right)$ be the natural coordinates of $\mathbb{R}^{4}$ and

$$
\alpha=-\bar{x}^{2} d \bar{x}^{1}+\bar{x}^{1} d \bar{x}^{2}-\bar{x}^{4} d \bar{x}^{3}+\bar{x}^{3} d \bar{x}^{4} .
$$

If $i: S^{3} \rightarrow \mathbb{R}^{4}$ is the natural immersion of the Euclidean 3-sphere in $\mathbb{R}^{4}$, the form $\eta=i^{*} \alpha$ defines an almost contact metric structure on $S^{3}$ which is moreover a Sasakian structure [3]. One can check (see [9]) that the homogeneous Sasakian structures on $S^{3}$ with respect to $\eta$ are those given in (29) after the isometry

$$
\varphi: \begin{aligned}
S^{3} & \longrightarrow S^{3} \\
\left(\bar{x}^{1}, \bar{x}^{2}, \bar{x}^{3}, \bar{x}^{4}\right) & \mapsto\left(\bar{x}^{1},-\bar{x}^{2},-\bar{x}^{3},-\bar{x}^{4}\right),
\end{aligned}
$$

namely

$$
\left(\bar{S}^{\lambda}\right)_{\bar{x}}=(1-\lambda) d \bar{x}^{2} \otimes d \bar{x}^{3} \wedge d \bar{x}^{4}-d \bar{x}^{3} \otimes d \bar{x}^{2} \wedge d \bar{x}^{4}+d \bar{x}^{4} \otimes d \bar{x}^{2} \wedge d \bar{x}^{3} .
$$

This homogeneous structures are obtained from the group of isometries

$$
G=\left\{\varphi \circ \Phi_{a} \circ \varphi^{-1} / a \in U(2)\right\}
$$

where $\Phi_{a}$ denotes the standard action of $U(2)$ on $S^{3}$. The subgroup

$$
H=\left\{\varphi \circ \Phi_{z} \circ \varphi^{-1} / z \in U(1)\right\}
$$

is a normal subgroup of $G$, where $z \in U(1)$ is seen in $U(2)$ as the matrix $\left(\begin{array}{cc}z & 0 \\ 0 & z\end{array}\right)$. Reduction by the action of $H$ gives the fibration

$$
\begin{aligned}
S^{3} & \rightarrow S^{2} \\
\left(z_{1}, z_{2}\right) & \mapsto\left(2 z_{1} z_{2},\left|z_{1}\right|^{2}-\left|z_{2}\right|^{2}\right)
\end{aligned}
$$


which is precisely the fibration given by the Sasakian structure $\eta$ in the sense of Theorem 5.1. The reduction described in Proposition 5.3 by the action of $H$ of the family of homogeneous structures (31) is (as we had in 4.2.1) the tensor $S=0$.

As for the second fibration, we take $\left(\bar{x}^{1}, \ldots, \bar{x}^{8}\right)$ the coordinates of $\mathbb{R}^{8}$ and

$$
\alpha=-\bar{x}^{2} d \bar{x}^{1}+\bar{x}^{1} d \bar{x}^{2}-\bar{x}^{4} d \bar{x}^{3}+\bar{x}^{3} d \bar{x}^{4}-\bar{x}^{6} d \bar{x}^{5}+\bar{x}^{5} d \bar{x}^{6}-\bar{x}^{8} d \bar{x}^{7}+\bar{x}^{7} d \bar{x}^{8} .
$$

The form $\eta=i^{*} \alpha$, where $i: S^{7} \rightarrow \mathbb{R}^{8}$ is the natural immersion of the Euclidean 7 -sphere, defines an almost contact metric structure on $S^{7}$ which is moreover Sasakian (cf. [3]). A homogeneous Sasakian structure on $S^{7}$ with respect to $\eta$ is obtained by transforming (30) with respect to the isometry

$$
\varphi: \begin{aligned}
S^{7} & \longrightarrow S^{7} \\
\left(\bar{x}^{1}, \ldots, \bar{x}^{8}\right) & \mapsto\left(\bar{x}^{1},-\bar{x}^{2}, \ldots,-\bar{x}^{8}\right),
\end{aligned}
$$

and reads

$$
\begin{aligned}
\bar{S}_{\bar{x}}= & -d \bar{x}^{3} \otimes d \bar{x}^{2} \wedge d \bar{x}^{4}+d \bar{x}^{4} \otimes d \bar{x}^{2} \wedge d \bar{x}^{3}-d \bar{x}^{5} \otimes d \bar{x}^{2} \wedge d \bar{x}^{6} \\
& +d \bar{x}^{6} \otimes d \bar{x}^{2} \wedge d \bar{x}^{5}-d \bar{x}^{7} \otimes d \bar{x}^{2} \wedge d \bar{x}^{8}+d \bar{x}^{8} \otimes d \bar{x}^{2} \wedge d \bar{x}^{7} .
\end{aligned}
$$

This family of homogeneous structure tensors are also obtained from the action of the group of isometries

$$
G=\left\{\varphi \circ \Phi_{a} \circ \varphi^{-1} / a \in U(4)\right\}
$$

where $\Phi_{a}$ denotes the standard action of $U(4)$ on $S^{7}$. The subgroup

$$
H=\left\{\varphi \circ \Phi_{z} \circ \varphi^{-1} / z \in U(1)\right\}
$$

is a normal subgroup of $G$, and reduction by the action of $H$ provides the fibration given by the Sasakian structure $\eta$ in the sense of Theorem 5.1. Again, the family (32) reduces to $S=0$.

A non trivial projection of homogeneous Sasakian structure tensors can be found in the following situation. Let $\pi: \bar{M} \rightarrow \mathbb{C} H(n)$ be a principal line bundle endowed with the Sasakian structure $(\phi, \xi, \eta, \bar{g})$ given by an invariant metric $\bar{g}$ and its corresponding mechanical connection $\eta$ in $\bar{M}$ (see [8]). Then, every homogeneous Kähler structure tensor $S$ in $\mathbb{C} H(n)$ can be obtained as the reduction of the Sasakian homogeneous structure tensor

$$
\bar{S}_{X^{H}} Y^{H}=\left(S_{X} Y\right)^{H}-\bar{g}\left(X^{H}, \phi Y^{H}\right) \xi, \quad \bar{S}_{X^{H}} \xi=-\phi X^{H}=\bar{S}_{\xi} X^{H}, \quad \bar{S}_{\xi} \xi=0,
$$

in the sense of Proposition 5.3. The description of all these tensors have been previously studied in 8 . Nevertheless, it is interesting to point out that the goal of that reference was the lift of structures from $\mathbb{C} H(n)$ to $\bar{M}$. The result given in Proposition 5.3 thus gives a reverse procedure of that particular situation. 


\section{Acknowledgements}

The authors are deeply indebted to Prof. Andrew Swann and Prof. P.M. Gadea for useful conversations about the topics of this paper.

This work has been partially funded by Ministerio de Ciencia e Innovación (Spain) under project MTM2011-22528.

\section{References}

[1] Abbena, E., Garbiero, S., Almost Hermitian homogeneous structures, Proc. Edinb. Math. Soc. (2) 31, 375-395 (1988).

[2] Ambrose, W., Singer, I.M., On homogeneous Riemannian manifolds, Duke Math. J. 25, 647-669 (1958).

[3] Blair, D. E., Riemannian geometry of contact and symplectic manifolds, Progress in Mathematics 203, Birkhäuser Boston, Inc., Boston, MA (2002).

[4] Castrillón López, M., Gadea, P.M., Swann, A.F., Homogeneous quaternionic Kähler structures and quaternionic hyperbolic space, Transform. Groups 11 no. 4, 575-608 (2006).

[5] Castrillón López, M., Gadea, P.M., Swann, A.F., Homogeneous structures on real and complex hyperbolic spaces, Illinois J. Math. 53 no. 2, 561-574 (2009).

[6] Fino, A., Intrinsic torsion and weak holonomy, Math. J. Toyama Univ. 21, 1-22 (1998),.

[7] Gadea P.M., Montesinos Amilibia, A., Muñoz Masqué, J., Characterizing the complex hyperbolic space by Kähler homogeneous structures, Math. Proc. Cambridge Philos. Soc. 128 no. 1, 87-94 (2000).

[8] Gadea P.M, Oubiña, J.A., Homogeneous Kähler and Sasakian structures related to complex hyperbolic spaces, Proc. Edinb. Math. Soc. (2) 53 no. 2, 393-413 (2010).

[9] Gadea, P.M., Oubiña, J.A., Homogeneous Riemannian Structures on Berger 3-spheres, Proc. Edinb. Math. Soc. 48, 375-387 (2005).

[10] Gadea, P.M., Oubiña, J.A., Reductive Homogeneous Pseudo-Riemannian Manifolds, Mh. Math. 124, 17-34 (1997).

[11] Kiričenko, V. F., On homogeneous Riemannian spaces with an invariant structure tensor, Sov. Math., Dokl. 21 , 734-737 (1980).

[12] Kobayashi, S., Nomizu, K., Foundations of Differential Geometry, John Wiley \& Sons, Inc. (Interscience Division), New York Volumes I \& II (1963, 1969). 
[13] Lee, J., Introduction to smooth manifolds, Graduate Texts in Mathematics, 218. Springer-Verlag, New York (2003).

[14] Marsden, J.E., Ostrowski, J., Symmetries in motion: geometric foundations of motion control, Nonlinear Sci. Today, 21 pp. (1996).

[15] Montgomery, R., Isoholonomic problems and some applications, Comm. Math. Phys. 128 no. 3, 565-592 (1990).

[16] Ogiue, K., On fiberings of almost contact manifolds, Ködai Math. Sem. Rep. 17 53-62 (1965).

[17] Salamon, S. M., Riemannian Geometry and Holonomy groups, Pitman Research Notes Math. 201, Longman (1989).

[18] Tricerri, F., Vanhecke, L., Homogeneous Structures on Riemannian Manifolds, Cambridge University Press, Cambridge (1983). 